\title{
Inference of field initialization
}

\author{
Technical Report UW-CSE-10-02-01 \\ University of Washington Department of Computer Science \& Engineering
} February 6, 2010

\author{
Fausto Spoto \\ Dipartimento di Informatica \\ Università di Verona, Italy \\ fausto.spoto@univr.it
}

\author{
Michael D. Ernst \\ Computer Science \& Engineering \\ University of Washington, USA \\ mernst@uw.edu
}

\begin{abstract}
A raw object is partially initialized, with only some of its fields set to legal values. A raw object may violate its object invariants, such as that a given field is non-null. Programs often need to manipulate partially-initialized objects, but they must do so with care. Furthermore, analyses must be aware of rawness. For instance, software verification cannot depend on object invariants for raw objects.

We present a static analysis that infers a safe over-approximation of the program variables, fields, or array elements that, at runtime, might hold non-fully initialized objects. Our formalization is flow-sensitive and considers the exception flow in the analyzed programs. We have proved the analysis to be sound.

We have also implemented our analysis, in a tool called JULIA that computes both nullness and rawness information. We have evaluated JULIA on over $50 \mathrm{~K}$ lines of code. We have compared its output to manually-written nullness and rawness information, and to an independently-written type-checking tool that checks nullness and rawness. JULIA's output is accurate and, we believe, useful both to programmers and to static analyses.
\end{abstract}

\section{Introduction}

Object-oriented programming languages, such as Java, allow the definition of instance variables or fields, that hold the state of the objects. Those fields are normally (but not necessarily) initialized inside the constructors defined in their class. Before that initialization, in Java, they hold the default value for their static type. For fields of reference type, the default value is null.

Many type-checking systems and static verification tools aim to prove that some object invariant, inv, holds for the objects of a given class. This means that inv must be true immediately after the object construction and must remain true every time a (public) method is entered or exited. A typical object invariant states that some field $f$ is non-null. This means that all constructors must initialize $f$ to a non-null value and that $f$ will never be reset to null later. This is an important piece of information, since it guarantees that no dereference of $f$ will ever throw a NullPointerException. This conclusion is not true inside the constructors. Before $f$ is initialized, it still holds the default value null. Our work focuses on initialization properties, rather than all ways any invariant can be violated, such as by setting a value to a non-null value that is inconsistent with its formal specification.

Since, in general, object invariants do not hold for partiallyinitialized ("raw" [9]) objects, it is important to identify those sites - program fields, parameters, return values, or array elements that might hold a raw object at run-time. These sites are not just the this variable inside the constructors: that variable can be passed to methods and stored in fields or arrays. Moreover, a raw variable

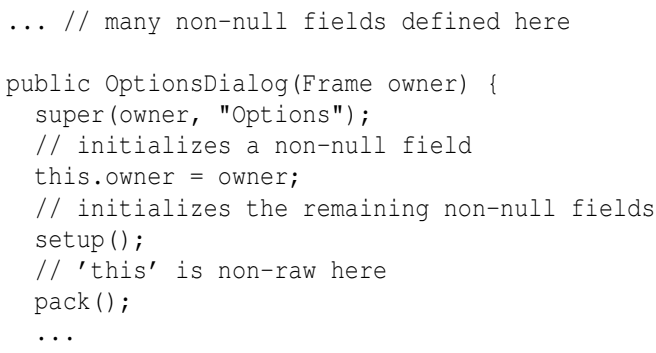

Figure 1: A snippet of code from the JFlex program.

loses its rawness as soon as all its fields have been initialized (or some relevant subset of its fields, see Section 5.4). Hence this might be non-raw inside a constructor, from a given program point onwards.

Figure 1 contains a snippet of code from class OptionsDialog of the JFlex scanner generator, one of the applications that we analyze in Section 6. Method setup () is called by the constructor of class OptionsDialog to help it build the object: this helper method initializes most of the fields of the object.

The JULIA tool performs our rawness analysis and infers that the receiver of setup () is raw, and all other references are nonraw, such as the receiver of pack (). Furthermore, JULIA infers that many fields are non-null. Without an inference of object initialization, a tool would either be unsound, or would be forced to conclude that all fields are possibly null. JULIA warns about any possibly-erroneous dereference; these include possibly-null fields wherever used in the program (unless the field can be proved to be non-null at that point), and non-null fields when used before the object is adequately initialized.

In principle, it would be correct to annotate all receivers, fields, parameters, and return values as @Raw: that would be a sound overapproximation of the set of raw sites. However, this would not be useful, and would hobble follow-on analyses [20] and human understanding. We aim for a precise analysis that infers as few rawness annotations as possible, only where needed.

We have extended the JULIA tool [16], which already contains a highly precise nullness analysis [22], to implement our rawness analysis. JULIA infers precise nullness and rawness annotations for non-trivial software, automatically, in a few minutes. We have also integrated JULIA with toolsets for working with annotations and pluggable type systems [2,4]. This enables us to compare our rawness analysis with pluggable type-checking for a nullness and rawness type system. The type system is weaker, but compositional and with easier-to-understand results. JULIA's results are correct by construction (the analyses in the JULIA tool are formally correct), but the type-checker still issues some warnings while type- 
checking them, because of the different perspective of the two tools. JULIA is based on flow and context-sensitive static analyses and abstract interpretation, while the checker framework is based on type-checking, augmented by flow-sensitivity and some other enhancements [20]. Nevertheless, we have achieved a good degree of integration of the two tools and Section 6 discusses the remaining differences and evaluates the quality of the automatic annotation w.r.t. that of a previous manual annotation that has been verified by type-checking.

Our work is relevant beyond the scope of nullness analysis. Our rawness analysis is not coupled to nullness analysis and is computed independently. It can be used in other contexts, whenever it is necessary to prove that some fields are definitely initialized at some program points. Moreover, the constraint-based analysis of Java bytecode in Section 5, and the structure of the correctness proof in Appendix A, can be used for other properties than rawness. Namely, JULIA embeds a constraint-based analysis, following the same scheme, that determines when arrays and collection classes are full, that is, contain non-null elements only (see Section 3).

This paper makes the following contributions:

- It defines and proves correct a rawness analysis for Java bytecode that is completely independent from any other analysis (such as nullness).

- It provides definitions and proofs that explicitly consider the exceptional flows in the program.

- We have implemented our rawness analysis, demonstrating its practicality. We have also integrated it with other nullness and rawness tools, permitting comparisons and increasing confidence in both toolsets.

- Experiments with our implementation demonstrate its precision. The experiments also yield insight into the algorithm's strengths and weaknesses and the properties of real code.

To the best of our knowledge, the first two points above are novel. For example, NIT's rawness inference is coupled to nullness and its theory does not consider exceptional flows [15]. Being independent from nullness analysis simplifies the formalization and the proof of correctness, and permits applicability to other problem domains. The last point above has never been investigated before. For example, NIT does not dump the rawness information that it computes, so its evaluation and its use for type-checking is impossible. See Section 2 for more comparisons with related work.

The rest of the paper is organized as follows. Section 2 overviews the most closely-related work. Section 3 reviews the nullness analysis implemented by the JULIA tool. Section 4 presents an operational semantics for Java bytecode, which is the concrete semantics of our abstract interpretation for rawness. Section 5 defines this constraint-based abstract interpretation. Section 6 reports on our experiments: we compared JULIA's output to manual annotations and to an independently-implemented type-checker. The proof of correctness is found in Appendix A. The JULIA tool is available for use through its web interface: http://julia.scienze.univr. it.

\section{Related work}

Fähndrich and Leino [9] check object initialization using a type qualifier (called "raw") that indicates how many fields are initialized. On exiting a constructor, the type is non-raw for that class and all of its superclasses, but still raw for any subtypes whose constructor has not yet been exited. In a raw type, all fields declared in that type are assumed to be possibly null, and the type checker enforces that these not-yet-initialized fields are not used. The nullness and rawness type-checker that we used in our experiments is a re-implementation of this algorithm, with enhancements. Delayed types [10] specify when fields can be assumed to have been initialized; by contrast, rawness specifies where fields can be assumed to have been initialized.

The most closely related work is NIT and JASTADD. NIT [15] is a nullness inference tool that, in parallel, also infers rawness. Unlike our work, their formalization and proofs do not consider exceptional flows. The NIT tool does not output any of the rawness annotations that it infers. JASTADD [6] infers nullness along with a coarser variant of rawness, in which each object is fully initialized or fully uninitialized, without reference to how many constructors have been exited. The rawness analysis of JASTADD is informally presented and is not proved correct. Rawness increased the percentage of references that JASTADD reports as safe from $69 \%$ to $71 \%$, for three packages in the JDK. In our experiments, JULIA reported over $98 \%$ of references to be safe. Like JULIA, NIT and JASTADD can produce an annotation file that can be inserted into Java source code or class files [2]. JACK [19] requires annotated method signatures, then does a flow-sensitive, alias-sensitive flow analysis to determine nullness and rawness types for local variables. It operates on bytecode. Like JASTADD, it infers the coarse version of rawness. Unlike JULIA, none of these tools' rawness analysis seems to have been evaluated and compared to manually-identified correct annotations.

Several other nullness inference tools for Java exist, but unlike JULIA they do not infer rawness annotations. DAIKON [8] runs the program and soundly outputs eNullable for variables that were ever observed to be null. It would be unsound to report @NonNull for values that were never observed to be null. DAIKON can produce an annotation file. HoudiNI [11] inserts @NonNull at every possible location, then runs a static checker. Whenever the static checker issues a warning, HoUDINI removes the relevant annotation. Houdini iterates this process until it reaches a fixed point. HOUDINI is neither sound nor complete. INAPA [7] is based on similar principles to HoudinI. FINDBUGS [14, 13] finds null pointer dereferences by using an imprecise analysis that produces many false warnings, but then prioritizing and filtering aggressively so that few false warnings are reported to a user. It attempts to infer programmer intent (w.r.t. nullness) based on code patterns. It is neither sound nor complete.

Our rawness analysis is a constraint-based abstract interpretation [5] of a concrete operational semantics for Java bytecode, presented in Section 4. Other operational semantics for Java bytecode are available, such as that of Freund and Mitchell [12]. Here we follow our formalization in [21], which is also the basis of the JULIA analyser [16], and hence we match theory with implementation. Our formalization is indebted to [17], where Java and Java bytecode are mathematically formalized and the compilation of Java into bytecode and its type-safeness are machine-proved. Our formalization of the state of the JVM (Definition 2 in Section 4.2) is similar to theirs, as well as our formalization of heap and objects.

\section{Nullness Inference}

The definition of our rawness analysis does not use any previous nullness analysis. Nevertheless, we use it here after a nullness analysis is performed to project its results (sets of initialized fields) over those fields that are deemed non-null by the nullness analysis, in order to infer rawness annotations that are useful for nullness typechecking (see Subsection 5.4). The exact nullness analysis which is used is not important here. We briefly describe the one implemented in the JULIA analysis tool.

In order to achieve a very high level of precision for nullness analysis, JULIA uses a mix of different techniques: static analyses based on denotational abstract interpretation and constraint-based 
abstract interpretation. Both come in different flavors, for inferring properties of the local variables, fields, arrays of references, and collections.

The kernel of the nullness analysis of JULIA is a denotational, bottom-up abstract interpretation of the bytecode, which builds logical formulas whose models over-approximate the nullness behaviors of the variables in scope in a piece of code. This analysis has been proved correct [25]. Since the number of variables, at a given program point, is necessarily finite, the Boolean formulas are finite and relatively small, so they can be efficiently represented by $b i$ nary decision diagrams [3]. The drawback of this approach is that it infers nothing about the nullness of the fields of the objects. Its precision is consequently unsatisfactory.

We now list three improvements, each of which increases precision but increases the computational cost. The overall system achieves high precision (see our results, presented in Section 6). A separate paper [22] presents all these techniques in detail and compares them w.r.t. precision and cost of the resulting nullness analyses.

(1) An optimistic approach [25] considers a field $f$, initialised by all constructors, to hold a non-null value (globally non- $n u I I$ ) unless a counter-example is found, that is, an assignment of a possibly null value to $f$. The resulting precision is slightly better than that of NIT [15], although it is in general slower.

(2) Local non-nullness [23] recognizes fields that are definitely non-null at specific program points, because they have just been assigned a non-null value or have been checked for non-nullness. Local non-nullness achieves a form of flow-sensitivity. The analysis is implemented as a denotational bottom-up abstract interpretation of the code.

(3) A constraint-based static analysis uses the same technique that we describe in Section 5, to identify full arrays and collection objects (hashsets, hashmaps, linked lists, etc.), whose elements are all non-null at some program points.

The output of a nullness analysis indicates, for every variable and every program point (where the variable is in scope), whether the variable may be null or is definitely non-null. This output can be used by a lint-like bug detection tool that informs the user of places where the null value might be dereferenced. (Most of the warnings will be false alarms, but a few might be actual bugs.) The tool output can also be inserted in the program for documentation and debugging, or can be exported to another tool to aid in further analysis. JULIA can produce null-dereference warnings directly, and can output nullness annotations to program source or other tools by using the Annotation File Utilities [2].

\section{Operational Semantics}

We describe here an operational semantics of the Java bytecode, that we abstract into our rawness analysis in Section 5 and prove correct in Appendix A.

\subsection{Syntax}

For simplicity of presentation, our formalism assumes int to be the only primitive type and classes to be the only reference types; we only allow instance fields and methods. Our implementation handles full sequential Java bytecode and all Java types. In particular, multithreading is not handled and the analysis of multithreaded applications might yield incorrect results, because JULIA assumes that immediately after a field is checked, it still has the same value.

We assume the Java bytecode is preprocessed into a control flow graph. This same representation is used in [21, 25, 24]; a similar representation is also chosen in [1], although, there, Prolog clauses encode the graph, while we work directly on the graph itself. A

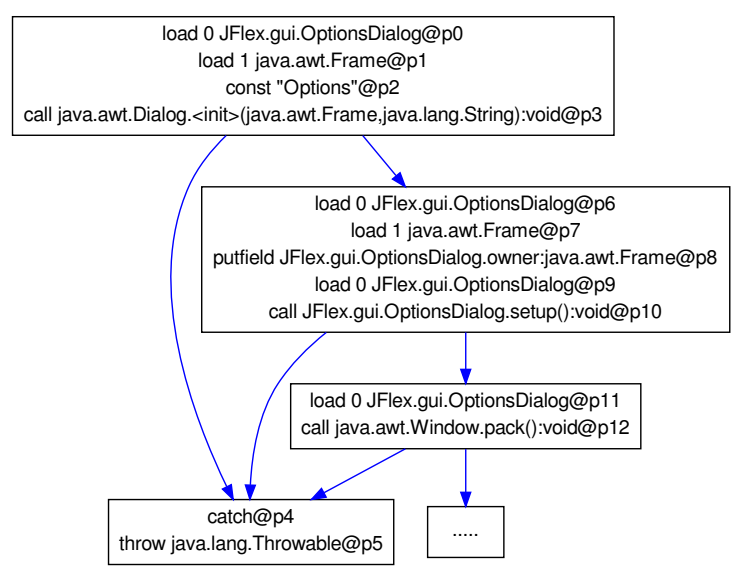

Figure 2: The blocks of code for the constructor in Figure 1.

control flow graph is a directed graph of basic blocks. All jumps are from the end of one basic block, to the beginning of another basic block. We graphically write

$$
\begin{gathered}
\text { ins @p } \\
\text { rest }
\end{gathered} \longrightarrow \begin{gathered}
b_{1} \\
\longrightarrow b_{m} \\
\hline
\end{gathered}
$$

for a block of code starting with a bytecode instruction ins at program point $p$, possibly followed by more bytecodes rest and linked to $m$ subsequent blocks $b_{1}, \ldots, b_{m}$. For instance, load 1 java.awt.Frame@p1 stands for an instance of the bytecode load 1 java.awt.Frame (which loads on the stack an object of class java. awt. Frame) occurring at program point $\mathrm{p} 1$. In most cases, the program point $p$ is irrelevant, so we write just ins. Bytecodes have explicit, inferred types.

Exception handlers start with a catch bytecode. A conditional bytecode, a virtual method call, or the selection of an exception handler, on the basis of the run-time type of the receiver or exception, is translated into a block linked to many subsequent blocks. Each subsequent block starts with a filtering bytecode, such as exception_is[_not] in the case of exceptional handlers, that specifies when that continuation is taken. They are not needed in Figure 1 since a default handler is used there: any kind of exception is caught and thrown back to the caller.

\subsection{Semantics}

Our operational semantics keeps a state, providing values for the variables of the program. An activation stack of states is used to model the method call mechanism, exactly as in an actual implementation of the JVM.

Definition 1. (Classes) The set of classes $\mathbb{K}$ in program $P$ is partially ordered w.r.t. $\leq$, which expresses the subclass relationship. A type is an element of $\mathbb{T}=\mathbb{K} \cup\{$ int $\}$. A class $\kappa \in \mathbb{K}$ has instance fields $\kappa . f: t$ (field $f$ of type $t \in \mathbb{T}$ defined in class $\kappa$ ), where $\kappa$ and $t$ are often omitted, and instance methods $\kappa . m(\vec{\tau}): t$ (method $m$ with arguments of type $\vec{\tau} \subseteq \mathbb{T}$, returning a value of type $t \in \mathbb{T} \cup\{$ void , defined in class $\kappa)$, where $\kappa, \vec{\tau}$, and $t$ are often omitted. Constructors are seen as methods named init and returning $\langle$ void $\rangle$.

\section{A state provides values to program variables.}

Definition 2. (State) A value is an element of $\mathbb{Z} \cup \mathbb{L} \cup\{$ nul1 , where $\mathbb{L}$ is an infinite set of memory locations. A state is a triple $\langle l\|s\| \mu\rangle$ where $l$ is an array of values (the local variables), $s$ a stack 
of values (the operand stack) which grows leftwards, and $\mu$ a memory, or heap, which binds locations to objects. The empty stack is written $\varepsilon$. An object $o$ belongs to class $o . \kappa \in \mathbb{K}$ (is an instance of $o . \kappa)$ and maps identifiers (the fields $f$ of class $o . \kappa$ and of its superclasses) into $o . f$, which can be a value or uninit. The set of states is $\Xi$. We write $\Xi_{i, j}$ when we want to fix the number $i$ of local variables and $j$ of stack elements. If $v$ is a value or uninit, we say that $v$ has type $t$ in a state $\langle l\|s\| \mu\rangle$ if $v \in \mathbb{Z} \cup\{$ uninit $\}$ and $t=$ int, or $v \in\{$ null, uninit $\}$ and $t \in \mathbb{K}$, or $v \in \mathbb{L}, t \in \mathbb{K}$ and $\mu(v) . \kappa \leq t$.

Compared to [21], Definition 2 allows fields to hold uninit. As will be clear from the formal semantics, this value is a special case of null or 0 that allows us to distinguish a field holding null or 0 because it has not been initialized yet, from a field already initialized, possibly to null or 0 .

Example 1. A possible state at the beginning of the constructor in Figure 2 is $\sigma=\left\langle\left[\ell, \ell^{\prime}\right]\|\varepsilon\| \mu\right\rangle$, where $\mu(\ell)$ (owner) $=$ uninit. Location $\ell$ contains the receiver $\mu(\ell)$ of the constructor, i.e., this, whose fields are not initialized at the beginning of the constructor. Location $\ell^{\prime}$ contains an object of class java. awt. Frame, the explicit argument of the constructor.

The JVM supports exceptions. Hence we distinguish normal states $\Xi$ arising during the normal execution of a piece of code, from exceptional states $\Xi$ arising just after a bytecode that throws an exception. States in $\underline{\Xi}$ have always a stack of height 1 containing a location (bound to the thrown exception object). We write them underlined in order to distinguish them from the normal states.

Definition 3. (JVM State) The set of JVM states (from now on just states) with $i$ local variables and $j$ stack elements is $\Sigma_{i, j}=$ $\Xi_{i, j} \cup \underline{\Xi}_{i, 1}$.

When we denote a state by $\sigma$, we do not specify if it is normal or exceptional. If we want to stress that we deal with a normal or with an exceptional state, then we write $\langle l\|s\| \mu\rangle$ in the first case and $\langle l\|s\| \mu\rangle$ in the second.

Example 2. A state $\sigma$ at the beginning of the block in Figure 2 containing catch@p4 might be an exceptional state arising when the call setup () aborts because of an OutofMemoryError (the code of that method contains many new statements). In that case, we would have $\sigma=\left\langle\left[\ell, \ell^{\prime}\right]\left\|\ell^{\prime \prime}\right\| \mu^{\prime}\right\rangle$, where $\ell$ and $\ell^{\prime}$ are as in Example $1, \mu^{\prime}(\ell)$ (owner) $=\ell^{\prime \prime \prime} \in \mathbb{L}$ (field owner of this has been already initialized at that point), $\mu\left(\ell^{\prime \prime}\right) . \kappa=$ OutOfMemoryError and $\mu\left(\ell^{\prime \prime}\right)$ has no uninit fields.

The semantics of a bytecode ins @ $p$ is a partial map ins : $\Sigma_{i_{1}, j_{1}} \rightarrow$ $\Sigma_{i_{2}, j_{2}}$ from an initial to a final state. The indices $i_{1}, j_{1}, i_{2}, j_{2}$ depend on $p$. The number and type of local variables and stack elements at each $p$ are statically known [18]. In the following we silently assume that the bytecodes are run in a program point with $i$ local variables and $j$ stack elements and that the semantics of the bytecodes is undefined for input states of wrong sizes or types. These assumptions are required by [18] and must hold for legal Java bytecode.

\subsection{Basic instructions}

Bytecode const $v$ pushes $v \in \mathbb{Z} \cup \mathbb{L} \cup\{$ null $\}$ on the stack. When $v \in \mathbb{L}$, location $v$ must be already allocated in the memory and hold an object of a very restricted set of classes, with all fields already initialized [18]. Formally, the semantics of this bytecode is

$$
\text { const } v=\lambda\langle l\|s\| \mu\rangle \cdot \begin{cases}\langle l\|v:: s\| \mu\rangle & \text { if } v \notin \mathbb{L} \text { or } \\ & (\mu(v) \text { is defined and has } \\ & \text { no uninit field) } \\ \text { undefined } & \text { otherwise. }\end{cases}
$$

The $\lambda$-notation defines a partial map, because of the undefined case. Namely, this bytecode is undefined when it tries to push a location which is not already in memory or has an uninitialized field. Since, above, $\langle l\|s\| \mu\rangle$ (where $s$ might be $\varepsilon$ ) is not underlined, the map is also undefined on exceptional states, i.e., the bytecode is executed when the JVM is not in an exceptional state. This is the case for all bytecodes but catch, which starts the exceptional handlers from an exceptional state.

Bytecode dup $t$ duplicates the top of the stack, of type $t$ :

$$
\text { dup } t=\lambda\langle l \| \text { top }:: s \| \mu\rangle .\langle l \| \text { top }:: \text { top }:: s \| \mu\rangle .
$$

Bytecode load it pushes on the stack the value of local variable number $i$, which must exist and have type $t$ :

$$
\text { load i } t=\lambda\langle l\|s\| \mu\rangle .\langle l\|l[i]:: s\| \mu\rangle \text {. }
$$

Conversely, bytecode store $i t$ pops the top of the stack of type $t$ and writes it in local variable $i$ :

$$
\text { store } i t=\lambda\langle l \| \text { top }:: s \| \mu\rangle .\langle l[i \mapsto t o p]\|s\| \mu\rangle \text {. }
$$

If $l$ contains less than $i+1$ variables, the resulting set of local variables gets expanded. The semantics of a conditional bytecode is undefined when its condition is false. For instance, if_ne $t$ checks if the top of the stack, which must have type $t$, is not 0 when $t=$ int or is not null otherwise:

if_ne $t=\lambda\langle l \|$ top $:: s \| \mu\rangle . \begin{cases}\langle l\|s\| \mu\rangle & \text { if top } \neq 0 \text { and top } \neq \text { null, } \\ \text { undefined } & \text { otherwise. }\end{cases}$

The undefined case corresponds to the fact that the JVM does not continue the execution of the code if the condition is false. Note that, in our formalization, conditional bytecodes are used in complementary pairs (for instance, if_ne and if_eq), at the beginning of the two branches of a condition, so that only one of them is defined for each given state.

\subsection{Object-manipulating instructions}

Some bytecodes create or access objects in memory. Bytecode new $\kappa$ pushes on the stack a reference to a new object $o$ of class $\kappa$, with reference fields initialized to uninit, that is, $o\left(\kappa^{\prime} \cdot f\right)=$ uninit for every field $\kappa^{\prime} . f: t$ with $t \in \mathbb{K}$ and $\kappa \leq \kappa^{\prime}$. Its semantics, new $\kappa$, is

$$
\lambda\langle l\|s\| \mu\rangle . \begin{cases}\langle l\|\ell:: s\| \mu[\ell \mapsto o]\rangle & \text { if there is enough memory, } \\ \underline{\langle l\|\ell\| \mu[\ell \mapsto o o m e]\rangle} & \text { otherwise }\end{cases}
$$

with $\ell \in \mathbb{L}$ fresh and oome new instance of OutofMemoryError. This is the first example of a bytecode that can throw an exception. Note that the initial value of the fields is fixed to uninit rather than to null or 0 , as it would be in a standard semantics [21].

The semantics getfield $\kappa . f: t$ of bytecode getfield $\kappa . f: t$ reads the field $\kappa . f: t$ of a receiver object rec popped from the stack, of type $\kappa$. It interprets uninit as null or 0 before pushing it on the stack, since the value uninit is not allowed on the stack (Definition 2). Hence getfield $\kappa . f: t$ is defined as

$$
\lambda\langle l\|r e c:: s\| \mu\rangle \cdot\left\{\begin{array}{lc}
\langle l \| \mu(\text { rec }) . f:: s \| \mu\rangle & \text { if } r e c \neq \text { null, } \mu(\text { rec }) . f \neq \text { uninit } \\
\langle l \| \text { null }:: s \| \mu\rangle & \text { if } r e c \neq \text { null, } \mu(\text { rec }) . f=\text { uninit } \\
& t \in \mathbb{K} \\
\langle l\|0:: s\| \mu\rangle & \text { if } \operatorname{rec} \neq \text { null, } \mu(\text { rec }) . f=\text { uninit } \\
& t=\text { int } \\
\langle l\|\ell\| \mu[\ell \mapsto n p e]\rangle & \text { otherwise }
\end{array}\right.
$$

with $\ell \in \mathbb{L}$ fresh and npe a new instance of NullPointerException. The bytecode putfield $\kappa . f: t$ writes the top of the stack, of type $t$, 
inside field $\kappa . f: t$ of the object pointed to by a value rec below the top of the stack, of type $\kappa$ ( $\ell$ and npe are as before). Its semantics putfield $\kappa . f: t$ is

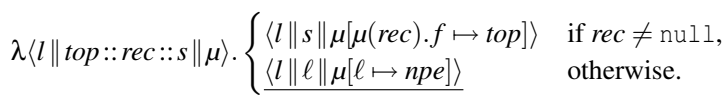

Note that this bytecode might only remove uninit from the approximation of field $f$, since the value top on the stack is not allowed to be uninit (Definition 2).

\subsection{Exception-handling instructions}

Bytecode throw $\kappa$ throws, explicitly, the object pointed by the top of the stack, of type $\kappa \leq$ Throwable ( $\ell$ and npe are as before):

$$
\text { throw } \kappa=\lambda\langle l \| \text { top }:: s \| \mu\rangle . \begin{cases}\frac{\langle l \| \text { top } \| \mu\rangle}{\langle l\|\ell\| \mu[\ell \mapsto n p e]\rangle} & \text { if } \text { if } \text { top }=\text { null } 1 .\end{cases}
$$

Bytecode catch starts an exception handler. It takes an exceptional state and transforms it into a normal state, subsequently used by the handler:

$$
\text { catch }=\lambda \underline{\langle l \| \text { top } \| \mu\rangle} .\langle l \| \text { top } \| \mu\rangle
$$

where top $\in \mathbb{L}$ has type Throwable. Note that catch is undefined on all normal states. After catch, bytecode exception_is $K$ selects the appropriate exception handler on the basis of the run-time class of top. Namely, it filters those states whose top of the stack is an instance of a class in $K \subseteq \mathbb{K}$. Its semantics exception_is $K$ is

$$
\lambda\langle l \| \text { top } \| \mu\rangle . \begin{cases}\langle l \| \text { top } \| \mu\rangle & \text { if } \text { top } \in \mathbb{L} \text { and } \mu(\text { top }) . \kappa \in K, \\ \text { undefined } & \text { otherwise. }\end{cases}
$$

Bytecode exception_is_not $K$ is just a shortcut for the bytecode exception_is $H$, where $H$ is the set of exception classes that are not an instance of some class in $K$.

\subsection{Method calls and return}

When a caller transfers the control to a callee $\kappa \cdot m(\vec{\tau}): t$, the JVM performs an operation makescope $\kappa \cdot m(\vec{\tau}): t$ which copies the topmost stack elements into the corresponding local variables and clears the stack.

Definition 4. Let $\kappa \cdot m(\vec{\tau}): t$ be a method or constructor and $\pi$ be the number of stack elements needed to hold its actual parameters, including the implicit parameter this. We define (makescope $\kappa . m(\vec{\tau})$ : $t): \Sigma \rightarrow \Sigma$ as

$$
\lambda\left\langle l\left\|v_{\pi-1}:: \cdots:: v_{1}:: r e c:: s\right\| \mu\right\rangle \cdot\left\langle\left[r e c, v_{1}, \ldots, v_{\pi-1}\right]\|\varepsilon\| \mu\right\rangle
$$

provided $r e c \neq$ null and the look-up of $m(\vec{\tau}): t$ from the class $\mu(r e c) . \kappa$ leads to $\kappa . m(\vec{\tau}): t$. We let it be undefined otherwise.

This formalizes the fact that the $i$ th local variable of the callee is a copy of the element located $(\pi-1)-i$ positions down the top of the stack of the caller.

Bytecode return $t$ terminates a method and clears its operand stack. If $t \neq \mathrm{void}$, the return value is the only element left on the final stack:

$$
\begin{aligned}
\text { return } \operatorname{void} & =\lambda\langle l\|s\| \mu\rangle .\langle l\|\varepsilon\| \mu\rangle \\
\text { return } t & =\lambda\langle l \| \text { top }:: s \| \mu\rangle .\langle l \| \text { top } \| \mu\rangle, \text { where } t \neq \text { void. }
\end{aligned}
$$

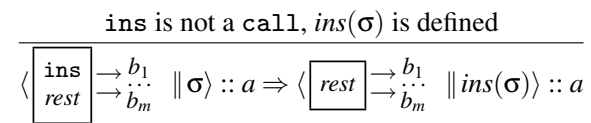

$\pi$ is the number of parameters of the target method, including this

$$
\begin{gathered}
\sigma=\left\langle l \| v_{\pi-1}:: \cdots:: v_{1}:: \text { rec }:: s \| \mu\right\rangle, \text { rec } \neq \text { null } \\
1 \leq i \leq n, \sigma^{\prime}=\left(\text { makescope } \kappa_{i} . m\right)(\sigma) \text { is defined } \\
f=\operatorname{first}\left(\kappa_{i} . m\right)
\end{gathered}
$$

$$
\left.\begin{array}{c}
\text { call } \kappa_{1} . m \ldots \kappa_{n} \cdot m \\
\text { rest }
\end{array} \rightarrow \underset{b_{m}}{b_{1}} \| \sigma\right\rangle:: a \Rightarrow\left\langle f \| \sigma^{\prime}\right\rangle::\left\langle\text { rest } \underset{\ddot{b}_{m}}{\rightarrow} \|\langle l\|s\| \mu\rangle\right\rangle:: a
$$

$\pi$ is the number of parameters of the target method, including this $\sigma=\left\langle l \| v_{\pi-1}:: \cdots:: v_{1}::\right.$ null $\left.:: s \| \mu\right\rangle$

$\ell \in \mathbb{L}$ is fresh and npe is a new instance of NullPointerException

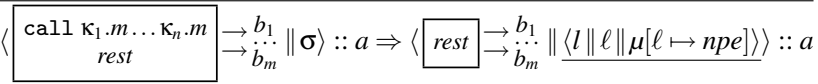

$$
\overline{\|} \|\langle\| \text { top } \| \mu\rangle\rangle::\left\langle b \|\left\langle l^{\prime}\left\|s^{\prime}\right\| \mu^{\prime}\right\rangle\right\rangle:: a \Rightarrow\left\langle b \|\left\langle l^{\prime} \| \text { top }:: s^{\prime} \| \mu\right\rangle\right\rangle:: a
$$

$$
\begin{aligned}
& \overline{\left\langle\square \| \underline{\langle l\|e\| \mu\rangle\rangle}::\left\langle b \|\left\langle l^{\prime}\left\|s^{\prime}\right\| \mu^{\prime}\right\rangle\right\rangle:: a \Rightarrow\left\langle b \| \underline{\left.\left\langle l^{\prime}\|e\| \mu\right\rangle\right\rangle}:: a\right.\right.} \\
& \frac{1 \leq i \leq m}{\left\langle\square \underset{\mathfrak{b}_{1}}{\longrightarrow} \| \sigma\right\rangle:: a \Rightarrow\left\langle b_{i} \| \sigma\right\rangle:: a}
\end{aligned}
$$

Figure 3: The transition rules of our operational semantics for Java bytecode (Section 4.7).

\subsection{The transition rules}

We can now define the operational semantics of our language.

Definition 5. A configuration is a pair $\langle b \| \sigma\rangle$ of a block $b$ and a state $\sigma$. It represents the fact that the JVM is going to execute $b$ in state $\sigma$. An activation stack is a stack $c_{1}:: c_{2}:: \cdots:: c_{n}$ of configurations, where $c_{1}$ is the topmost, current or active configuration.

The operational semantics of a Java bytecode program is a relation between activation stacks. It models the transformation of the activation stack induced by the execution of each single bytecode.

Definition 6. The (small step) operational semantics of a Java bytecode program $P$ is a relation $a^{\prime} \Rightarrow_{P} a^{\prime \prime}$ ( $P$ is usually omitted) providing the immediate successor activation stack $a^{\prime \prime}$ of an activation stack $a^{\prime}$. It is defined by the rules in Figure 3.

Rule 1 executes an instruction ins, different from call, by using its semantics ins. The JVM then moves forward to run the rest of the instructions. Rule 2 calls a method on a non-null receiver. It looks up the correct implementation $\kappa_{i} \cdot m(\vec{\tau}): t$ of the method, by using the look-up procedures of the language, and finds the block $b$ where that implementation starts. It then builds its initial state $\sigma^{\prime}$, by using makescope, and creates a new current configuration containing $b$ and $\sigma^{\prime}$. It pops the actual arguments from the old current configuration and the call from the instructions still to be executed at return time. Since a method call can actually call many implementations, depending on the run-time class of the receiver, this rule is apparently non-deterministic. However, only one thread of execution will continue, since we assume that the method lookup rules of the language are deterministic (as in Java bytecode). Control returns to the caller by rule 4 , which rehabilitates the configuration of the caller but forces the memory to be that at the end of the execution of the callee. The return value of the callee is pushed on the stack of the caller. This rule is executed if the state reached at the end of the caller is a normal state. If it is an exceptional state, rule 5 is executed instead, which propagates the exception back to the caller. If a method call occurs on a null receiver, 


$$
\begin{aligned}
& \left\langle\begin{array}{c}
\text { load 0 0D@p0 } \\
\text { load 1 java.awt.Frame@p1 } \\
\text { const"Options"@p2 } \\
\text { call java.awt.Dialog.〈init }\rangle \ldots \text {...p3 }
\end{array} \mid \rightarrow b_{6} \|\left\langle\left[\ell, \ell^{\prime}\right]\|\varepsilon\| \mu\right\rangle\right\rangle:: a
\end{aligned}
$$

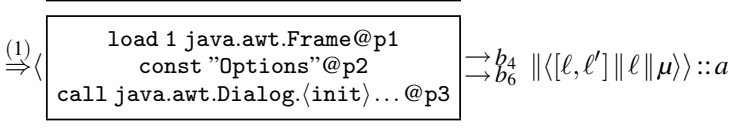

$$
\begin{aligned}
& \stackrel{(1)}{\Rightarrow}\left\langle\begin{array}{c}
\text { const "Options"@ } 22 \\
\text { call java.awt.Dialog. }\langle\text { init }\rangle \ldots @ \mathrm{p} 3
\end{array} \rightarrow b_{6} \|\left\langle\left[\ell, \ell^{\prime}\right]\left\|\ell^{\prime}:: \ell\right\| \mu\right\rangle\right\rangle:: a \\
& \stackrel{(1)}{\Rightarrow}\left\langle\text { call java.awt.Dialog. }\langle\text { init }\rangle \ldots @ \mathrm{p} 3 \rightarrow b_{4} \|\left\langle\left[\ell, \ell^{\prime}\right]\left\|\ell^{\prime \prime}:: \ell^{\prime}:: \ell\right\| \mu\right\rangle\right\rangle:: a \\
& \stackrel{(2)}{\Rightarrow}\left\langle\text { first } \|\left\langle\left[\ell, \ell^{\prime}, \ell^{\prime \prime}\right]\|\varepsilon\| \mu\right\rangle\right\rangle::\left\langle\square \underset{\square}{\rightarrow} b_{6} \quad \|\left\langle\left[\ell, \ell^{\prime}\right]\|\varepsilon\| \mu\right\rangle\right\rangle:: a \\
& \stackrel{(\ldots)}{\Rightarrow}\left\langle\square \|\left\langle\left[\ell, \ell^{\prime}, \ell^{\prime \prime}\right]\|\varepsilon\| \mu^{\prime}\right\rangle\right\rangle::\left\langle\square \rightarrow b_{6} \|\left\langle\left[\ell, \ell^{\prime}\right]\|\varepsilon\| \mu\right\rangle\right\rangle:: a \\
& \stackrel{(4)}{\Rightarrow}\left\langle\square \underset{\square}{\rightarrow} b_{6} \quad \|\left\langle\left[\ell, \ell^{\prime}\right]\|\varepsilon\| \mu^{\prime}\right\rangle\right\rangle:: a \\
& \stackrel{(6)}{\Rightarrow}\left\langle\begin{array}{c}
\text { load 0 0D@p6 } \\
\text { load 1 java.awt.Frame@p7 } \\
\text { putfield 0D.owner...@p8 } \\
\text { load 0 0D@p9 } \\
\text { call 0D.setup() : void@p10 }
\end{array} \rightarrow b_{4} \quad \|\left\langle\left[\ell, \ell^{\prime}\right]\|\varepsilon\| \mu^{\prime}\right\rangle\right\rangle:: a \\
& \stackrel{(1)}{\Rightarrow}\left\langle\begin{array}{c}
\text { load } 1 \text { java.awt.Frame@p7 } \\
\text { putfield OD.owner...@p8 } \\
\text { load 0 0D@p9 } \\
\text { call 0D.setup() : void@p10 }
\end{array} \rightarrow \rightarrow b_{11} \quad \|\left\langle\left[\ell, \ell^{\prime}\right]\|\ell\| \mu^{\prime}\right\rangle\right\rangle:: a \\
& \stackrel{(1)}{\Rightarrow}\left\langle\begin{array}{c}
\text { putfield OD.owner...@p8 } \\
\text { load 0 OD@p9 } \\
\text { call oD.setup }() \text { : void@p10 }
\end{array} \rightarrow b_{4} \quad \|\left\langle\left[\ell, \ell^{\prime}\right]\left\|\ell^{\prime}:: \ell\right\| \mu^{\prime}\right\rangle\right\rangle:: a \\
& \stackrel{(1)}{\Rightarrow}\left\langle\begin{array}{c}
\text { load 0 0D@p9 } \\
\text { call 0D.setup }(): \text { void@p10 }
\end{array} \rightarrow b_{11}^{4} \|\langle\left[\ell, \ell^{\prime}\right]\|\varepsilon\| \underbrace{\mu^{\prime}\left[\mu(\ell) \text {.owner } \mapsto \ell^{\prime}\right]}_{\mu^{\prime \prime}}\rangle\right\rangle:: a \\
& \stackrel{(1)}{\Rightarrow}\left\langle\text { call OD.setup() : void@p10 } \underset{\rightarrow}{\rightarrow} b_{11} \|\left\langle\left[\ell, \ell^{\prime}\right]\|\ell\| \mu^{\prime \prime}\right\rangle\right\rangle:: a
\end{aligned}
$$

Figure 4: A partial execution according to the semantics of Figure 3. $b_{x}$ is the block in Figure 2 starting at $\mathrm{p} x$. OD stands for JFlex.gui.OptionsDialog. Location $\ell^{\prime \prime}$ points to the string "Options" from the constant pool. first is the first block of the constructor of java.awt.Dialog. $\stackrel{(\ldots)}{\Rightarrow}$ is a complete execution of the latter and $\mu^{\prime}$ is the memory at its end.

rule 3 creates a new state whose stack contains only a reference to a NullPointerException. No actual call happens in this case. Rule 6 applies when all instructions inside a block have been executed; it runs one of its immediate successors, if any. This rule is normally deterministic, since if a block of our formalization of the Java bytecode has two or more immediate successors then they start with mutually exclusive conditional instructions and only one thread of control is actually followed.

From now on, when we use the notation $\Rightarrow$, we often specify the rule in Figure 3 which is used at each derivation step; for instance, we write $\stackrel{(1)}{\Rightarrow}$ for a derivation step through rule $(1)$.

Example 3. Consider the state $\left\langle\left[\ell, \ell^{\prime}\right]\|\varepsilon\| \mu\right\rangle$ from Example 1 at $\mathrm{p} 0$ in Figure 2. The operational semantics can proceed from $\mathrm{p} 0$ as in Figure 4.

\section{Rawness Analysis}

JULIA collects rawness information with a constraint-based rawness analysis which is performed at the end and independently from the nullness analysis. The rawness analysis builds a constraint: a graph whose nodes contain sets of fields that have not been initialized yet. There is a node for each local variable at a given program point and for each method parameter and return value. There is also a node for each field and another for the values stored into arrays; differently from local variables, fields and arrays have flowinsensitive approximations. Assignments and parameter passing are modeled as directed arcs $a \rightarrow b$ in the graph. They represent the inclusion of the content of $a$ in the content of $b$.

When an object is created by a new $\kappa$ bytecode, all fields of $\kappa$ and of its superclasses are used as an approximation for the newly created object, left on top of the stack, since its has no initialized field at that point. Then arcs are built to link subsequent program points. For instance, the arc $l_{0} @ \mathrm{p} 0 \rightarrow l_{0} @ \mathrm{p} 1$ is built for the program in Figure 1. But the arc $l_{0} @ \mathrm{p} 0 \rightarrow s_{0} @ \mathrm{p} 1$ is also built, since local variable 0 at $\mathrm{p} 0$ is loaded on the stack as its only element $s_{0}$ at $\mathrm{p} 1$. Arcs are built for all possible flows of control induced by loops, conditionals and exceptions. For instance, in Figure 1, both arcs $l_{0} @ \mathrm{p} 3 \rightarrow l_{0} @ \mathrm{p} 4$ and $l_{0} @ \mathrm{p} 3 \rightarrow l_{0} @ \mathrm{p} 6$ are built. Assignments to fields give rise to arrows from the rightvalue to the node for the field. For instance, the assignment at $\mathrm{p} 8$ induces the arc: $s_{1} @ \mathrm{p} 8 \rightarrow$ owner. In order to model the fact that field owner is now initialized, a filtering arc is built also, for each definite alias of the receiver. For instance, the same assignment at $\mathrm{p} 8$ introduces the arc $l_{0} @ \mathrm{p} 8 \stackrel{\text { owner }}{\rightarrow} l_{0} @ \mathrm{p} 9$ instead of $l_{0} @ \mathrm{p} 8 \rightarrow l_{0} @ \mathrm{p} 9$. The former arc states that all fields in $l_{0} @ \mathrm{p} 8$ are included in $l_{0} @ \mathrm{p} 9$, but owner. The definite aliasing information needed here is the same used, for instance, in our nullness analysis (see [25]), so we can recycle it.

When all arcs have been built for the whole program, the constraint is solved through a least fixpoint calculation. The approximation of each variable is an over-approximation of the set of its fields that might not have been initialized yet. For instance, in Figure 1, the approximation computed for $l_{0} @ \mathrm{p} 0$ is the set $S=$ $\{$ owner,...\} of all fields of class OptionsDialog and of its superclasses, and that for $s_{0} @ \mathrm{p} 10$ is $S \backslash\{$ owner $\}$, since the non-null field owner has been already initialized at $\mathrm{p} 10$. The approximation computed for $s_{0} @ \mathrm{p} 12$ is $\emptyset$, since all fields of class OptionsDialog and of its superclasses have been already initialized at $\mathrm{p} 12$.

\subsection{The abstraction map for rawness}

In order to formalize our rawness analysis and prove its correctness, we define now the abstraction map from states to rawness.

Definition 7. (Rawness Abstraction) Let $\sigma=\left\langle\left[v_{0} \ldots v_{i-1}\right] \| w_{j-1}\right.$ : $\left.\cdots:: w_{0} \| \mu\right\rangle$ be a state (possibly underlined) with $i$ local variables and $j$ stack elements. Its rawness abstraction $\alpha(\sigma)$ maps the symbols $\left\{l_{0} \ldots l_{i-1}, s_{0} \ldots s_{j-1}, f_{1}, \ldots, f_{n}\right\}$, where $f_{1}, \ldots, f_{n}$ are all the fields in $P$, into sets of uninitialized fields, that is, fields that have not been initialized yet for each local variable, stack element or field:

$$
\begin{aligned}
& \alpha(\sigma)\left(l_{k}\right)= \begin{cases}\emptyset & \text { if } v_{k} \in \mathbb{Z} \cup\{\text { null }\} \\
\left\{f \mid \mu\left(v_{k}\right) \cdot f=\text { uninit }\right\} & \text { if } v_{k} \in \mathbb{L}\end{cases} \\
& \alpha(\sigma)\left(s_{k}\right)= \begin{cases}\emptyset & \text { if } w_{k} \in \mathbb{Z} \cup\{\text { nul }\} \\
\left\{f \mid \mu\left(w_{k}\right) \cdot f=\text { uninit }\right\} & \text { if } w_{k} \in \mathbb{L}\end{cases} \\
& \alpha(\sigma)\left(f_{k}\right)=\left\{f \mid \text { there exists } \ell \in \mathbb{L} \text { s.t. } \mu\left(\mu(\ell) \cdot f_{k}\right) \cdot f=\text { uninit }\right\} .
\end{aligned}
$$

By Definition 7, the rawness of a stack element or local variable is the set of fields still bound to uninit in the object they hold. The rawness of a field $f_{k}$, instead, includes a field $f$ if there is an object $\mu(\ell)$ at $\ell$ with field $f_{k}$ bound to a location $\ell^{\prime}=\mu(\ell) \cdot f_{k}$ that holds an object $\mu\left(\ell^{\prime}\right)$ whose field $f$ is still uninit. Note that we silently 
assume that $\mu\left(\mu(\ell) . f_{k}\right) . f$ is well defined, i.e., that all its components are defined. Instance fields are flattened by this abstraction, i.e., they are treated as static fields and we cannot distinguish fields with the same name but in different objects. This abstraction is necessary to get a finite static analysis, since the number of objects in memory is potentially unbound.

Example 4. Consider the state $\sigma=\left\langle\left[\ell, \ell^{\prime}\right]\|\varepsilon\| \mu\right\rangle$ from Example 1. Its abstraction is such that owner $\in \alpha(\sigma)\left(l_{0}\right)$.

\subsection{The abstract constraint}

A program $P$ induces a constraint, which is a graph whose nodes contain a set of uninitialized fields. There are many kinds of nodes:

- $l_{k} @ p$ stands for the $k$ th local variable $(k \geq 0)$ at program point $p$

- $s_{k} @ p$ stands for the $k$ th stack element $(k \geq 0)$ at program point $p$

- $f @ e w$ stands for field $f$, at any program point

- return@m stands for the return value of method $m$

- exception@m stands for any exception thrown by method $m$

- $l_{k} @$ end of $m$ stands for the $k$ th local variable $(k \geq 0)$ at the end of every normal execution of method $m$

- $\left\{\kappa_{1}, f_{1}, \ldots, \kappa_{n} . f_{n}\right\}$ stands for a node containing those uninitialized fields

Arcs are directed: $n_{1} \rightarrow n_{2}$ states that all the fields in $n_{1}$ are also in $n_{2}$. The filtering arc $n_{1} \stackrel{f}{\rightarrow} n_{2}$ states that all the fields in $n_{1}$ except $f$ are also in $n_{2}$. Arcs are built for each pair of subsequent bytecode instructions:

Definition 8. Let ins $p @ p$ and ins $q @ q$ be two bytecodes. Let $i_{p}$ and $j_{p}$ be the number of local variables and stack elements at the beginning of the execution of ins ${ }_{p}$, respectively. Let $U_{i_{p}, j_{p}}=$ $\left\{l_{k} @ p \rightarrow l_{k} @ q \mid 0 \leq k<i_{p}\right\} \cup\left\{s_{k} @ p \rightarrow s_{k} @ q \mid 0 \leq k<j_{p}\right\}$. We define the constraints con(ins in $_{p}$, ins $\left.q @ q\right)$ as follows. If ins $q$ is not a catch then:

$$
\begin{aligned}
& \operatorname{con}\left(\text { const } v, \text { ins }_{q}\right)=\operatorname{con}\left(\operatorname{catch}, \text { ins }_{q}\right) \\
& \left.=\text { con }(\text { exception_is[_not }] K, \text { ins }_{q}\right)=U_{i_{p}, j_{p}} \\
& \operatorname{con}\left(\operatorname{dup} t, \text { ins }_{q}\right)=U_{i_{p}, j_{p}} \cup\left\{s_{j_{p}-1} @ p \rightarrow s_{j_{p}} @ q\right\} \\
& \operatorname{con}\left(\text { load } x t, \text { ins }_{q}\right)=U_{i_{p}, j_{p}} \cup\left\{l_{x} @ p \rightarrow s_{j_{p}} @ q\right\} \\
& \text { con }\left(\text { store } x t, \text { ins }_{q}\right)=\left\{l_{k} @ p \rightarrow l_{k} @ q \mid 0 \leq k<i_{p}, k \neq x\right\} \\
& \cup\left\{s_{k} @ p \rightarrow s_{k} @ q \mid 0 \leq k<j_{p}-1\right\} \cup\left\{s_{j_{p}-1} @ p \rightarrow l_{x} @ q\right\} \\
& \text { con(if_ne } t, \text { ins }_{q} \text { ) }=U_{i_{p}, j_{p}-2} \\
& \operatorname{con}\left(\text { new } \kappa, \text { ins }_{q}\right)=U_{i_{p}, j_{p}} \cup\left\{\left\{\kappa^{\prime} \cdot f: t \mid t \in \mathbb{K} \text { and } \kappa \leq \kappa^{\prime}\right\} \rightarrow s_{j_{p}} @ q\right\} \\
& \operatorname{con}\left(\text { getfield } f, \text { ins }_{q}\right)=U_{i_{p}, j_{p}-1} \cup\left\{f @ e w \rightarrow s_{j_{p}-1} @ q\right\} \\
& \operatorname{con}\left(\text { putfield } f, \text { ins }_{q}\right)=\left\{l_{k} @ p \rightarrow l_{k} @ q \mid 0 \leq k<i_{p},\left(l_{k}, s_{j_{p}-2}\right) \notin \text { alias }_{p}\right\} \\
& \cup\left\{s_{k} @ p \rightarrow s_{k} @ q \mid 0 \leq k<j_{p}-2,\left(s_{k}, s_{j_{p}-2}\right) \notin \operatorname{alias}_{p}\right\} \\
& \cup\left\{l_{k} @ p \stackrel{f}{\rightarrow} l_{k} @ q \mid 0 \leq k<i_{p},\left(l_{k}, s_{j_{p}-2}\right) \in \text { alias }_{p}\right\} \\
& \cup\left\{s_{k} @ p \stackrel{f}{\rightarrow} s_{k} @ q \mid 0 \leq k<j_{p}-2,\left(s_{k}, s_{j_{p}-2}\right) \in \operatorname{alias}_{p}\right\} \\
& \cup\left\{s_{j_{p}-1} @ p \rightarrow f @ e w\right\} \\
& \operatorname{con}\left(\operatorname{call} m_{1} \ldots m_{n}, \text { ins }_{q}\right)=\cup_{k=1}^{n} \cup_{u=0}^{\pi-1}\left\{s_{j_{p}-u-1} @ p \rightarrow l_{\pi-u-1} @ \operatorname{first}\left(m_{k}\right)\right\} \\
& \cup\left\{\text { return@ } m_{k} \rightarrow s_{j_{p}-\pi} @ q \mid 1 \leq k \leq n\right\} \\
& \cup\left\{\begin{array}{l|l}
l_{k} @ p \rightarrow l_{k} @ q & \begin{array}{l}
1 \leq k<i_{p} \text { and if }\left(l_{k}, s_{j_{p}-u-1}\right) \in \text { alias }_{p} \\
\text { for some } 0 \leq u<\pi \text { then at least an } m_{h} \\
\text { contains a store } l_{\pi-u-1} t
\end{array}
\end{array}\right\} \\
& \cup\left\{\begin{array}{l|l}
l_{\pi-u-1} @ \text { end of } m_{w} \rightarrow l_{k} @ q & \begin{array}{l}
1 \leq k<i_{p}, 1 \leq w \leq n, \\
\left(l_{k}, s_{j_{p}-u-1} \in \text { alias }_{p}\right. \\
\text { for some } 0 \leq u<\pi \text { and no } m_{h} \\
\text { contains a store } l_{\pi-u-1} t
\end{array}
\end{array}\right\}
\end{aligned}
$$

$$
\begin{aligned}
& \cup\left\{\begin{array}{l|l}
s_{k} @ p \rightarrow s_{k} @ q & \begin{array}{l}
1 \leq k<j_{p}-\pi \text { and if }\left(s_{k}, s_{j_{p}-u-1}\right) \in \text { alias }_{p} \\
\text { for some } 0 \leq u<\pi \text { then at least an } m_{h} \\
\text { contains a store } l_{\pi-u-1} t
\end{array}
\end{array}\right\} \\
& \cup\left\{\begin{array}{l|l}
l_{\pi-u-1} @ \text { end of } m_{w} \rightarrow s_{k} @ q & \begin{array}{l}
1 \leq k<j_{p}-\pi, 1 \leq w \leq n, \\
\left(s_{k}, s_{j_{p}-u-1} \in \text { alias }_{p}\right. \\
\text { for some } 0 \leq u<\pi \text { and no } m_{h} \\
\text { contains a store } l_{\pi-u-1} t
\end{array}
\end{array}\right\} .
\end{aligned}
$$

If, instead, ins $q$ is a catch, we define

$$
\begin{aligned}
& \text { con }(\text { throw K, catch })=\left\{l_{k} @ p \rightarrow l_{k} @ q \mid 0 \leq k<i_{p}\right\} \\
& \cup\left\{s_{j_{p}-1} @ p \rightarrow s_{0} @ q\right\} \\
& \text { con }\left(\text { call } m_{1} \ldots m_{n}, \text { catch }\right)= \cup_{k=1}^{n} \cup_{u=0}^{\pi-1}\left\{s_{j_{p}-u-1} @ p \rightarrow l_{\pi-u-1} @ f i r s t\left(m_{k}\right)\right\} \\
& \cup\left\{\text { exception@ } m_{k} \rightarrow s_{0} @ q \mid 1 \leq k \leq n\right\} \cup\left\{l_{k} @ p \rightarrow l_{k} @ q \mid 1 \leq k<i_{p}\right\} \\
& \text { con }\left(\text { ins }_{p}, \text { catch }\right)= U_{i_{p}, 0}, \text { where ins } s_{p} \text { is not a throw nor a call. }
\end{aligned}
$$

Moreover, if $p$ is a program point inside method $m$, we define the constraints

$$
\begin{aligned}
\text { final_con }(\text { throw } \kappa)= & \left\{s_{j_{p}-1} @ p \rightarrow \text { exception } @ m\right\} \\
\text { final_con }(\text { return void })= & \left\{l_{k} @ p \rightarrow l_{k} @ \text { end of } m \mid 0 \leq k<i_{p}\right\} \\
\text { final_con }(\operatorname{return} t)= & \left\{l_{k} @ p \rightarrow l_{k} @ \text { end of } m \mid 0 \leq k<i_{p}\right\} \\
& \cup\left\{s_{j_{p}-1} @ p \rightarrow \text { return@ } m\right\}
\end{aligned}
$$

where $t \neq$ void.

Definition 8 has two cases. The first is when ins $q$ is not a catch. This means that the normal output state of ins ${ }_{p}$ flows to the beginning of ins $q$. If ins $p$ is a const, the sets of uninitialized fields for local variables and stack elements do not change. This is also the case for catch, exception_is and exception_is_not. Hence we build a constraint $U_{i_{p}, j_{p}}$ that states this fact. For dup, we also build an arc saying that the set of uninitialized fields for the new top of the stack $\left(s_{j_{p}} @ q\right)$ contains all the uninitialized fields of the old top of the stack $\left(s_{j_{p}-1} @ p\right)$. Similar constraints are built for load and store: the latter keeps the approximation of the local variables unchanged but for $l_{x}$ that gets the approximation of the old top of the stack. If ins $p$ is an if_ne, two elements are removed from the stack. If it is a new $\kappa$, the new top of the stack contains all fields defined in $\kappa$ or in a superclass $\kappa^{\prime}$ of $\kappa$, since they are not yet initialized. Bytecodes getfield and putfield create arcs from and to the node $f @ e w$ for the accessed field $f$. The latter bytecode modifies the rawness of every definite alias of its receiver $s_{j_{p}-2}$, since field $f$ is being initialized. The constraints generated when ins $p$ is a call are the most complex. They link the actual arguments (the topmost stack elements of the caller) to the formal arguments (the lowest local variables at the first bytecode first $\left(m_{k}\right)$ of the initial block of each callee $m_{k}$ ). Moreover, the local variables $l_{k}$ of the caller and its stack elements $s_{k}$ that are not actual arguments might keep their approximation or can see it improved when they are a definite alias of an actual argument $s_{j_{p}-u-1}$ and the corresponding formal argument $l_{\pi-u-1}$ is not updated inside the callee. If this is the case, then the final approximation for $l_{\pi-u-1}$ inside the callee can be used as approximation for $l_{k}$ (respectively, $s_{k}$ ) after the call. This situation is important since it allows helper functions to improve the rawness approximation for the variables of the caller. This is the case, for instance, of setup () in Figure 1, whose code initializes tens of fields of an OptionsDialog.

The second case of Definition 8 is when ins $q$ @ is a catch. This means that ins $\mathrm{s}_{p}$ is executed, it throws an exception $e$ which is caught by ins $q$ and stored as $s_{0} @ q$. In any case, the original rawness approximation for the local variables remains correct. If ins $_{p}$ is not a call nor a throw, then $e$ is an internal exception [18] 
without uninitialized fields, so we can use $U_{i_{p}, 0}$. Otherwise, $e$ might be the top of the stack $\left(s_{j_{p}-1} @ p\right)$ for throw or an exception thrown by the called method(s) for call. If ins $p$ is a call, we also link the actual arguments to the formal ones.

The function final_con generates constraints for the final bytecode of a block with no successors. That bytecode can only be a throw or a return inside some method $m$. In the first case the top of the stack $\left(s_{j_{p}-1} @ p\right)$ is linked to the exception thrown by $m$. In the second case it is linked to the return value of the method, if any, and the local variables are linked to the approximation of the local variables at the end of $m$.

Example 5. Consider ins $p=\operatorname{load} 1$ java.awt.Frame@p1 and ins $_{q}=$ const "Options" @p2 from Figure 1. At p1 we have $i_{p}=$ 2 local variables and $j_{p}=1$ stack elements. Thus $\operatorname{con}\left(\right.$ ins $_{p}$, ins $\left.\mathbf{s}_{q}\right)=$ $\left\{l_{0} @ \mathrm{p} 1 \rightarrow l_{0} @ \mathrm{p} 2, l_{1} @ \mathrm{p} 1 \rightarrow l_{1} @ \mathrm{p} 2, l_{1} @ \mathrm{p} 1 \rightarrow s_{1} @ \mathrm{p} 2, s_{0} @ \mathrm{p} 1 \rightarrow\right.$ $\left.s_{0} @ \mathrm{p} 2\right\}$.

Example 6. Let ins $\mathrm{s}_{p}=\mathrm{call}$ java.awt.Dialog. $\langle$ init $\rangle \ldots \mathrm{p} 3$ and ins $\mathrm{s}_{q}=$ load 0 JFlex.gui.OptionsDialog@p6 from Figure 1 At p3 we have $i_{p}=2$ local variables and $j_{p}=3$ stack elements. Our aliasing analysis computes alias $_{\mathrm{p} 3}=\left\{\left(l_{0}, s_{0}\right),\left(l_{1}, s_{1}\right)\right\}$. This call has $n=1$ targets and $\pi=3$ parameters (including the implicit this parameter). Let first be the first bytecode in the initial block of the constructor $m$ of java.awt.Dialog, whose code does not contain any store 0 nor any store 1 . Hence $\operatorname{con}\left(\right.$ ins $_{p}$, ins $\left.\mathbf{s}_{q}\right)=$ $\left\{s_{0} @ \mathrm{p} 3 \rightarrow l_{0} @\right.$ first, $s_{1} @ \mathrm{p} 3 \rightarrow l_{1} @$ first, $s_{2} @ \mathrm{p} 3 \rightarrow l_{2} @$ first, $l_{0} @$ end of $m \rightarrow l_{0} @ \mathrm{p} 6, l_{1} @$ end of $\left.m \rightarrow l_{1} @ \mathrm{p} 6\right\}$.

We can define the constraints induced by the whole program.

Definition 9. Let $\begin{gathered}\text { ins }_{1} \\ \cdots \\ \text { ins }_{n}\end{gathered} \rightarrow \begin{aligned} & b_{1} \\ & \rightarrow \ddot{b}_{m}\end{aligned}$ be a block. If $m>0$, its induced constraints are $\cup_{k=1}^{n-1} \operatorname{con}\left(\right.$ ins $_{k}$, ins $\left.s_{k+1}\right) \cup \cup_{h=1}^{m} \operatorname{con}\left(\right.$ ins $_{n}$, first $\left.\left(b_{h}\right)\right)$, where first $\left(b_{h}\right)$ is the first instruction in $b_{h}$. If $m=0$, they are $\cup_{k=1}^{n-1} \operatorname{con}\left(\right.$ ins $_{k}$, ins $\left._{k+1}\right) \cup$ final_con $\left(\right.$ ins $\left._{n}\right)$. Those induced by a program $P$ are the union of the constraints induced by each block of $P$.

\subsection{Correctness of the analysis}

Once the constraints for $P$ have been built, they can be solved, i.e., a least solution can be found, satisfying the inclusions represented by their arcs. This is possible since arcs (normal and filtering) stand for monotonic functions from the approximation of their source to that of their sink. Hence a least solution exists, is unique, and can be computed, for instance, with an iterated fixpoint calculation from the empty approximation for each node.

Definition 10. The solution of a constraint $G$ is the least assignment $\mathcal{S}$ of sets of fields to nodes, such that $\mathcal{S}\left(\left\{f_{1}, \ldots, f_{n}\right\}\right)=$ $\left\{f_{1}, \ldots, f_{n}\right\}$ for every node $\left\{f_{1}, \ldots, f_{n}\right\} \in G, \mathcal{S}\left(n_{1}\right) \subseteq \mathcal{S}\left(n_{2}\right)$ for every $n_{1} \rightarrow n_{2} \in G$ and $\mathcal{S}\left(n_{1}\right) \backslash\{f\} \subseteq \mathcal{S}\left(n_{2}\right)$ for every $n_{1} \stackrel{f}{\rightarrow} n_{2} \in$ $G$.

Example 7. The solution of the constraints generated for the program in Figure 1 is such that $\mathcal{S}\left(l_{0} @ \mathrm{p} 0\right)=\{$ owner,$\ldots\}$ contains all the fields defined in OptionsDialog and in its superclasses. Moreover, owner $\notin \mathcal{S}\left(s_{0} @ \mathrm{p} 10\right) \neq \emptyset$ and $\mathcal{S}\left(s_{0} @ \mathrm{p} 12\right)=\emptyset$ (that is, all fields of OptionsDialog and of its superclasses have been definitely assigned when calling pack ()).

We can now provide the correctness result for our analysis. It states that the abstraction of all the states generated during the exe- cution of $P$ according to our operational semantics is over-approximated by the solution of the constraint generated for $P$. The hypothesis of this proposition guarantees that the considered execution is feasible, i.e., it did not hang the Java Virtual Machine.

Proposition 1. Let $\left\langle b_{\text {first }(\text { main })} \| \varsigma\right\rangle \Rightarrow^{*}\left\langle\begin{array}{c}\begin{array}{c}\text { ins@ } p \\ \text { rest }\end{array} \rightarrow b_{1} \rightarrow \\ \rightarrow b_{m}\end{array} \| \sigma\right\rangle:: a$ be any execution of our operational semantics, from method ma in and an initial state $\varsigma$ whose objects in memory have no uninitialized fields, with ins $(\sigma)$ defined when ins is not a call, or with $\sigma \in \Xi$ with at least $\pi$ stack elements when ins is a call with $\pi$ parameters. Let there be $i$ local variables and $j$ stack elements at $p$. Then for every $0 \leq k<i$ we have $\alpha(\sigma)\left(l_{k}\right) \subseteq \mathcal{S}\left(l_{k} @ p\right)$, for every $0 \leq k<j$ we have $\alpha(\sigma)\left(s_{k}\right) \subseteq \mathcal{S}\left(s_{k} @ p\right)$ and for every field $f_{k}$ we have $\alpha(\sigma)\left(f_{k}\right) \subseteq S\left(f_{k} @ e w\right)$.

Note that, in Java bytecode, method ma in receives an array of strings as parameter and those strings have no uninitialized fields. Hence the hypothesis on $\varsigma$ is sensible.

Proposition 1 is proved in Appendix A.

\subsection{Building the ${ }^{\mathrm{R} a w}$ annotations}

Our rawness analysis can be used whenever one wants to know if some field of a given variable is definitely initialized at a given program point. As we said in Section 3, this is the case for nullness analysis, since existing type-checkers for nullness allow the specification of a set of non-nul fields, which are always initialized by all constructors of their defining class and are always assigned a non-null value. Hence they do hold a non-null value, but only after their first initialization. Where this is not yet the case, rawness must be specified.

Given a set of non-null fields $N N$, our analysis infers a superset of the variables $v$ at a given program point $p$ and a superset of the fields $f$ of the program that should be typed as @Raw. It is enough to check if $\mathcal{S}(v @ p) \cap N N \neq \emptyset$ or $\mathcal{S}(f @ e w) \cap N N \neq \emptyset$, respectively. Similarly for the formal parameters of the methods and for their return value. It is also possible to derive class-specific rawness, that is, to determine if all non-null fields defined in a given class $\kappa$ have been initialized or not. Namely, a superset of the variables $v$ at a given program point $p$ and a superset of the fields $f$ of the program that should be typed as @Raw $(\kappa)$ can be determined by checking if $\mathcal{S}(v @ p) \cap\{\kappa \cdot g \mid \kappa \cdot g \in N N\} \neq \emptyset$ or $\mathcal{S}(f @ e w) \cap\{\kappa \cdot g \mid$ $\kappa . g \in N N\} \neq \emptyset$, respectively.

\section{Experimental results}

We have implemented the analysis that is defined in Section 5 and proved correct in Appendix A. This section describes experiments that assess the effectiveness of the analysis. The key question is whether JULIA's output is correct.

\subsection{Subject programs and analysis output}

We applied the inference tool, JULIA, to three programs. JFlex 1.4.3 is a scanner generator (http:// jflex.de/). Plume is library of utility programs and data structures (http: // code.google.com/ p/plume-lib/, downloaded on Feb. 3, 2010). The Annotation File Utilities (AFU) 3.0 are tools for reading/writing Java annotations [2].

Figure 5 lists the sizes of the programs, the analysis time, and raw data about Julia's output.

JULIA's scalability depends on the size of the reachable code in an application, rather than on the lines of source code. JULIA starts its analysis at all entry points to the program, and then proceeds to discover and analyze all reachable code in the program. It treats as entry points: (1) any public static void main (String[]) 


\begin{tabular}{|r|r|r|r|r|r|r|r|r|r|}
\hline & \multicolumn{2}{|c|}{ size } & \multicolumn{2}{|c|}{ reachable program \& libraries } & \multicolumn{2}{c|}{ analysis time (sec.) } & \multicolumn{2}{c|}{ dereferences } & \multicolumn{2}{c|}{ inferred annotations } \\
program & (lines) & methods & lines & bytecodes & nullness & rawness & safe / all (\%) & @NonNull & eRaw \\
\hline \hline JFlex & 14987 & 3885 & 39097 & 390315 & 282 & 3 & $8584 / 8751(98.1)$ & $572 / 741(77.2)$ & $6 / 1109(0.5)$ \\
\hline AFU & 13892 & 4246 & 38415 & 412332 & 346 & 3 & $5054 / 5143(98.3)$ & $642 / 854(75.2)$ & $15 / 1124(1.3)$ \\
\hline plume & 19652 & 5400 & 49921 & 511277 & 660 & 4 & $8481 / 8613(98.5)$ & $617 / 915(67.4)$ & $3 / 1118(0.3)$ \\
\hline plume progs & 6167 & 5400 & 49921 & 511277 & 660 & 4 & $6048 / 6148(98.4)$ & $235 / 300(78.4)$ & $3 / 339(0.9)$ \\
\hline
\end{tabular}

Figure 5: Experimental results. "Lines" is counted with the cloc program (http://cloc. sourceforge. net/). Size is computed separately for the application as downloaded, and for the reachable, analyzed portion of the program, including any reachable libraries but not counting unreachable methods in the program or the libraries. Analysis time and results are for the most precise nullness analysis currently available in JULIA. Dereferences are counted only in the the reachable application code (which does not include libraries). Safe dereferences are those that JULIA can guarantee will never throw a null pointer exception at run time. In the "Inferred annotation" columns, the denominator is the total number of sites at which the annotation could possibly be written, in fields and method signatures of the reachable application code. The percentage of inferred annotations is also given. The last row reports the analysis of plume, as in the previous line, but with statistics projected over the 10 classes that have a main () method; see Section 6.2.2.

method, and (2) any public static void test*() method in a class that extends TestCase, to handle JUnit tests.

The rawness analysis is fast - just a few seconds. The nullness analysis runs as long as 11 minutes on our subject programs. Most of the nullness analysis runtime is due to the aliasing and shape analysis that it calls as a subroutine.

Recall from Section 1 the two primary uses for a nullness/rawness inference: to indicate locations where a null pointer exception may be thrown, or to provide annotations for a human or a follow-on analysis. The last two groups of columns in figure 5 address these two uses.

A "dereference" is any location at which a variable must be nonnull to avoid throwing a null pointer exception. These include field and method dereferences, array accesses, array length expressions, throw statements, and synchronization operations. JULIA proves that over $98 \%$ of the dereferences in each application program are safe - that is, these locations can never throw a null pointer exception at run time. This fact can aid in optimization and reasoning. For comparison, these numbers are around $80 \%$ in the case of NIT (see [23] for a comparison).

Figure 5 indicates the number of annotations inferred, and the maximum number of sites at which the annotation could possibly be inferred. For eNonNull, the sites include fields, method formal parameters, and method return types. A single type may have multiple sites; for example, up to three @NonNull annotations could be placed on Map<String, Ob ject $>$. Receivers and constructor results are not counted as sites, because they are trivially non-null. Primitive and void types are never counted, because they cannot be null. The sites for @Raw are the same as those for @NonNull, plus receivers. Constructor results are trivially non-raw.

JULIA annotates a significant amount of the program, lessening the programmer burden. (Either eNonNull annotations, or a smaller but still significant number of @Nullable annotations, are automatically inserted into the program source code.) @Raw is inferred for as much as $1.3 \%$ of all references in the program.

Our experiments count annotations on fields and in method signatures. These are the places that annotations are most useful. It would also be possible to infer types for local variables or for expressions within a method body, but our experiments ignore this possibility, and JULIA does not perform such output.

Depending on the program, JULIA proves that $67-78 \%$ of all references can be marked as @NonNull. Furthermore, only a very few references are marked as @Raw.

Previous evaluations of nullness inference tools have generally reported numbers like these, quantifying the tool's output. A problem with such numbers is that they do not indicate whether the tool's output is correct or useful. Section 6.2 addresses the correctness question by comparing JULIA's output to manual annotations of nullness and rawness. Section 6.3 addresses usefulness of both the nullness and rawness annotations, for one particular use: proving a program is free of null pointer dereferences, or fixing any such problems in the program.

\subsection{Comparison to human-written annotations}

This section compares JULIA's annotations to a correct set of annotations written by a human.

As part of a different project, plume was previously annotated with nullness and rawness annotations. ${ }^{1}$ The manual annotations use @NonNull as the default (except for local variables, which default to @Nullable), and so only write an annotation for @Nullable references. This leads to fewer annotations overall. Plume has 508 nullness or rawness annotations on 312 distinct lines, plus another 36 warning suppression annotations.

The manual annotations were checked by a pluggable type-checker built upon the Checker Framework [20]. The type-checker verified both the correctness of the annotations, and that there are no in plume. The outcome of this process is a guarantee that the annotations are correct, and that plume has no null pointer dereferences. ${ }^{2}$ This gives perspective on the $132(=8613-8481)$ possibly-unsafe dereferences that Julia reports in plume: they are probably all false warnings.

To gain perspective on these false warnings and on JULIA's strengths and weaknesses, both of this paper's authors examined differences between the manual annotations and JULIA's output. We examined rawness annotations in all of plume, and nullness annotations in a subset of plume.

\subsubsection{Rawness comparison for all of plume}

We examined all rawness differences, everywhere in plume. Plume as downloaded contains 7 instances of @Raw, and JULIA's output contains 3 instances of @Raw. One @Raw annotation is in both sets, so there are 8 differences to examine.

Three manual @Raw annotations, on method receivers, are not inferred by JULIA and are extraneous - they are weaknesses in the manual annotation. The program type-checks with these annotations, but it also type-checks without them, so there is no need for them. They were inserted at a time when the type-checker did need them, because it was unable to infer that an object is initialized before its constructor exits. JULIA and the current type-checker can make such inferences. These annotations have since been removed from plume.

\footnotetext{
${ }^{1}$ Except for this section, all of our experiments use a version of plume from which all nullness/rawness annotations have been removed.

${ }^{2}$ The guarantee is modulo the fact that when the programmer annotated the program, the programmer also suppressed some type-checking warnings. The programmer only did so when manual reasoning indicated the warning was a false warning, but the programmer may have made mistakes during this manual reasoning.
} 


\begin{tabular}{|c|c|c|c|c|c|c|}
\hline \multirow{2}{*}{$\begin{array}{l}\text { Inconse- } \\
\text { quential }\end{array}$} & \multirow{2}{*}{\begin{tabular}{|c|}
$\mathrm{T}-\mathrm{C}$ \\
artifact
\end{tabular}} & \multirow{2}{*}{$\begin{array}{c}\text { Unanalyzed } \\
\text { code }\end{array}$} & \multicolumn{2}{|c|}{ Manual } & \multicolumn{2}{|r|}{ Julia } \\
\hline & & & error & weaknes & err & weakness \\
\hline 21 & 40 & 69 & 8 & 18 & 2 & 48 \\
\hline IMWS LA & SW BO & DCUC UL & SUME & UN MW & $\mathrm{JE}$ & RRESIMAIT JW \\
\hline 1344 & 1822 & $\begin{array}{lll}32 & 34 & 3\end{array}$ & 2 & 16 & 2 & $\begin{array}{llllll}0 & 8 & 6 & 4 & 4 & 6\end{array}$ \\
\hline
\end{tabular}

Figure 6: Number of lines of differences between manual annotations and JULIA output, classified according to Section 6.2.2. In general, each difference results in two lines of diff output. The table classifies the same differences twice: once into coarser and once into finer-grained categories.

Three manual @Raw annotations on parameters of type Object are not inferred by JULIA, because JULIA does more detailed analysis than the type-checker. In particular, JULIA recognizes that any value passed to the given methods is initialized up to the @object constructor, even if it is not be fully initialized. The type-checker requires these parameters to be marked @Raw because they are not fully initialized.

Finally, two @Raw annotations inferred by JULIA are a result of imprecision in JULIA's analysis.

\subsubsection{Full comparison for programs in plume}

We examined all differences between the manual annotations and JULIA's output, for a subset of plume. For our subset, we chose all the programs in plume: each class that contains a main method. There are 10 such classes (out of 44), and they contain about 1/4 of plume's lines of code. Running the diff program on these classes yields 206 lines of differences (compared to 1450 lines of differences for all of plume). The last line of Figure 5 provides more measurements.

Usually, there are 2 lines of diff output per difference: one line in the diff output shows the old code, and one shows the new code. In some cases, such as import statements and warning suppression, there are more or fewer. To permit counting without fear of ambiguity, we always use number of lines of diff output.

We classified each of the 206 lines of diff output according to the following categories:

\section{Inconsequential differences}

IM (import statement) JULIA added an import statement that is redundant with an existing one.

WS (whitespace) The difference is in whitespace or the order of modifiers.

LA (@LazyNonNull) The manual annotation uses @LazyNonNull, which means that the variable may start out as null, but once set to a non-null value, it may continue to be reassigned but is never again assigned to null. JULIA uses @Nullable, which is equivalent from JULIA's point of view.

\section{Type-checker artifacts}

SW (@SuppressWarnings) The difference is the removal of a @SuppressWarnings annotation that was present in the original program, in a method body. It was placed there to overcome a limitation of the type-checker. JULIA may or may not have a similar limitation; if it does, that will be reflected in a difference in some field or method signature.

BO (inside method body) The difference is an annotation within a method body (other than @SuppressWarnings), which our experiment ignores but the type-checker needs.

\section{Unanalyzed code}

DC (dead code) A method is never executed, so JULIA does not bother to annotate it. For example, this applies to old code that is no longer used, and to code used for debugging. As another example that alone accounts for 25 of the 32 lines of diff, an API required a Reader object, but did not use it, so plume created a DummyReader class and passed that to the API.

UC (unreachable code) JULIA cannot find a path that executes a given method, because no such path exists in plume, though a path may exist in client code. The prime cause for this is the fact that one of the selected classes, EntryReader, is really a library, not a program. Its main method is just a simple usage example that does not fully exercise the class.

UL (unanalyzed library) JULIA cannot find a path to a method because the path starts in a library that JULIA did not analyze. For example, a class's boolean start (RootDoc doc) method is called reflectively when Javadoc executes, but JULIA does not add Javadoc's main method to the set of entry points for every program.

\section{Errors in manual annotations}

SU (static initialization unchecked) The type checker verifies that instance fields are properly initialized by the time the constructor exits, but does not do a similar check for static fields, so a static field marked as @NonNull may contain null. The plume authors have subsequently verified these errors and corrected them by changing the annotation to @Nullable.

ME (manual annotation error, other) A constructor argument was improperly marked as @NonNull that should have been @Nullable. This annotation has also now been fixed. Overall, JULIA did not reveal any null pointer errors, only the $4(=8 / 2)$ incorrect annotations.

Weaknesses in manual annotations

UN (unannotated code) The developers did not consider this code worth annotating: for example, the code is undocumented, is used for testing, or is under development. JULIA's inference results would make the annotation task much easier.

MW (manual annotation weakness, other) The programmer wrote @Nullable on a method return value that is actually always non-null. The annotation type-checks, but the more precise annotation is better.

Errors in Julia output

JE (errors in JULIA annotations) JULIA uses multiple annotations for arrays of references and for generic classes of the standard Java library - one annotation for the container and one for the element type. However, JULIA does not yet do so for userdefined generic classes such as $\operatorname{Pair}\langle\mathrm{S}, \mathrm{T}\rangle$.

Weaknesses in JULIA output

PR (property) Method System.getProperty () always returns nonnull for certain properties, such as "java.class.path" and "line.separator" — unless the program does something perverse like System.getProperties (). remove ("java. class.path");, which is not the case here. But JULIA does not infer this fact.

RE (regular expressions) A call to Matcher.group () returns nonnull if it is guarded by a call to Matcher.matches (), and the string representation contains parentheses at the top level. This is beyond JULIA's reasoning capabilities.

SI (static initialization) A static field was initialized to non-null at the beginning of main (), and the class was never used outside the dynamic scope of the main () method. JULIA cannot guarantee that fact about its use, and so marked the field as @Nullable. The field was not set in the static initializer because the initializer has no access to the arguments to main.

MA (map) A map was queried via get (), using a value that came from an iterator that was indirectly related to the map. Thus, get () returned a map value and not null, but JULIA did not recognize this fact.

IT (iterator) In a class that implements java.util. Iterator, a superclass constructor somewhere in a library uses a (differ- 
ent) iterator. JULIA was not able to establish that the two iterators are not aliased.

JW (JULIA weaknesses, other) JULIA cannot establish that the result of TimeZone.getDisplayName() is non-null. (Nor can we, but the Javadoc implies that it is.) As a more complicated example, CalendarBuilder.build () sets a calendar value to null, apparently to satisfy Java's definite assignment check, but then calls parser.parse () which is guaranteed (by the structure of its input) to eventually either throw an exception or make a callback that will set the variable that CalendarBuilder.build() eventually returns.

Suppose that a programmer wants to use a type-checker to verify that an unannotated version of the 10 plume programs has no null pointer errors. Further suppose that the libraries those programs use are already annotated. (The type-checker comes with an annotated version of the JDK and some other libraries.) The programmer can start with JULIA's output, then edit approximately $75(=(40+69+$ $2+48) / 2$ ) lines. This modest cost demonstrates that JULIA's output is accurate and can be useful to programmers.

\subsection{Type-checking of inferred annotations}

JULIA infers most of the necessary nullness and rawness annotations, which is a great help to a programmer who would otherwise be forced to write them all from scratch.

Section 6.2 compared JULIA's annotations to a correct set of annotations. Another comparison would be to count the number of type-checking errors that occur when type-checking a program with and without JULIA's annotations. We used the Nullness Checker that is distributed with the Checker Framework [20, 4]. This typechecker requires the programmer to annotate the program, then verifies the correctness of the annotations and the absence of null pointer errors. It shares no code with the JULIA inference tool, making it an effective cross-validation for the correctness of both tools.

Unfortunately, the number of type-checking errors is not a good metric of annotation quality. In general, adding a correct, necessary annotation may increase or decrease the number of compiler warnings, depending on how the variable is used and the correctness and verifiability of the surrounding annotations. For instance, for all of plume (not just the programs as evaluated in Section 6.2), here is the number of type-checking warnings:

\begin{tabular}{|c|l|}
\hline Warnings & Annotations \\
\hline 365 & no annotations \\
408 & JULIA nullness annotations only \\
411 & JULIA nullness \& rawness annotations \\
\hline
\end{tabular}

As the annotations get more complete and closer to what a programmer needs in order to verify that the program has no null pointer errors, the number of warnings increases! Therefore, we do not believe this is a useful metric, for evaluating annotations or suggesting fixes to them.

\section{Conclusion}

We have defined a new analysis for computing field initialization ("rawness"), proved it correct, and implemented it. Our experiments compare it to human-provided and machine-checked correct annotations, and these experiments confirm the accuracy of the analysis.

\section{References}

[1] E. Albert, P. Arenas, S. Genaim, G. Puebla, and D. Zanardini. Cost analysis of Java bytecode. In ESOP, pages 157-172, Mar. 2007.
[2] Annotation File Utilities website. http:

//types.cs.washington.edu/annotation-file-utilities/, Feb. 3, 2010.

[3] R. E. Bryant. Graph-based algorithms for boolean function manipulation. IEEE Trans. Comput., 35(8):677-691, 1986.

[4] Checker Framework website. http://types.cs.washington.edu/checker-framework/, Feb. 3, 2010.

[5] Patrick Cousot and Radhia Cousot. Abstract interpretation: a unified lattice model for static analysis of programs by construction or approximation of fixpoints. In POPL, pages 238-252, 1977.

[6] Torbjörn Ekman and Görel Hedin. Pluggable checking and inferencing of non-null types for Java. J. Object Tech., 6(9):455-475, Oct. 2007.

[7] Arnout F. M. Engelen. Nullness analysis of Java source code. Master's thesis, University of Nijmegen Dept. of Computer Science, Aug. 102006.

[8] Michael D. Ernst, Jeff H. Perkins, Philip J. Guo, Stephen McCamant, Carlos Pacheco, Matthew S. Tschantz, and Chen Xiao. The Daikon system for dynamic detection of likely invariants. Science of Computer Programming, 69(1-3):35-45, Dec. 2007.

[9] Manuel Fähndrich and K. Rustan M. Leino. Declaring and checking non-null types in an object-oriented language. In OOPSLA, pages 302-312, Nov. 2003.

[10] Manuel Fähndrich and Songtao Xia. Establishing object invariants with delayed types. In OOPSLA, pages 337-350, Oct. 2007.

[11] Cormac Flanagan, Rajeev Joshi, and K. Rustan M. Leino. Annotation inference for modular checkers. Information Processing Letters, 2(4):97-108, Feb. 2001.

[12] Stephen N. Freund and John C. Mitchell. A type system for object initialization in the Java bytecode language. ACM TOPLAS, 21(6):1196-1250, Nov. 1999.

[13] David Hovemeyer and William Pugh. Finding more null pointer bugs, but not too many. In PASTE, pages 9-14, June 2007.

[14] David Hovemeyer, Jaime Spacco, and William Pugh. Evaluating and tuning a static analysis to find null pointer bugs. In PASTE, pages 13-19, Sep. 2005.

[15] Laurent Hubert, Thomas Jensen, and David Pichardie. Semantic foundations and inference of non-null annotations. In FMOODS, pages 132-149, 2008.

[16] Web interface to the JULIA analyzer. http://julia.scienze.univr.it.

[17] G. Klein and T. Nipkow. A machine-checked model for a Java-like language, virtual machine, and compiler. ACM TOPLAS, 28(4):619-695, 2006.

[18] Tim Lindholm and Frank Yellin. The Java Virtual Machine Specification. Addison-Wesley, Reading, MA, USA, 2nd edition, 1999.

[19] Chris Male and David J. Pearce. Non-null type inference with type aliasing for Java. http://www.mcs.vuw.ac.nz/ djp/files/MP07.pdf, Aug. 20, 2007.

[20] Matthew M. Papi, Mahmood Ali, Telmo Luis Correa Jr., Jeff H. Perkins, and Michael D. Ernst. Practical pluggable types for Java. In ISSTA, pages 201-212, July 2008.

[21] É. Payet and F. Spoto. Magic-sets transformation for the analysis of Java bytecode. In SAS, pages 452-467, Aug. 2007.

[22] F. Spoto. The nullness analyser of JULIA. Submitted for publication, 2010.

[23] F. Spoto. Precise null-pointer analysis. Software and Systems Modeling, 2010. To appear.

[24] F. Spoto, F. Mesnard, and É. Payet. A termination analyser for Java bytecode based on path-length. ACM TOPLAS, 2010. To appear.

[25] Fausto Spoto. Nullness analysis in boolean form. In SEFM, Nov. 2008.

\section{A. Proofs}

The following lemma states the correctness of the solution of the constraints generated for a program $P$, w.r.t. the execution of a single bytecode. It will be used in the proof of Proposition 1 in 
Section A.1.

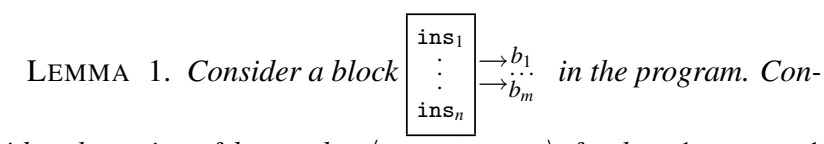

sider the pairs of bytecodes $\left\langle\right.$ ins $_{k}$, ins $\left._{k+1}\right\rangle$ for $k=1, \ldots, n-1$

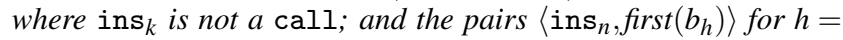
$1, \ldots, m$ where ins $\mathbf{s}_{n}$ is not a call. Name those pairs, generically, $\left\langle\right.$ ins $_{p} @ p$, ins $\left._{q} @ q\right\rangle$. Let $\sigma_{p}$ be such that both $\sigma_{q}=$ ins $p\left(\sigma_{p}\right)$ and ins $q\left(\sigma_{q}\right)$ are defined. Let there be $i_{p}$ local variables and $j_{p}$ stack elements at $p$ and $i_{q}$ local variables and $j_{q}$ stack elements at q. If $\alpha\left(\sigma_{p}\right)\left(l_{k}\right) \subseteq \mathcal{S}\left(l_{k} @ p\right)$ for every $0 \leq k<i_{p}, \alpha\left(\sigma_{p}\right)\left(s_{k}\right) \subseteq$ $S\left(s_{k} @ p\right)$ for every $0 \leq k<j_{p}$ and $\alpha\left(\sigma_{p}\right)\left(f_{k}\right) \subseteq \mathcal{S}\left(f_{k} @ e w\right)$ for all fields $f_{k}$, then we have $\alpha\left(\sigma_{q}\right)\left(l_{k}\right) \subseteq \mathcal{S}\left(l_{k} @ q\right)$ for every $0 \leq k<$ $i_{q}, \alpha\left(\sigma_{q}\right)\left(s_{k}\right) \subseteq \mathcal{S}\left(s_{k} @ q\right)$ for every $0 \leq k<j_{q}$ and $\alpha\left(\sigma_{q}\right)\left(f_{k}\right) \subseteq$ $\mathcal{S}\left(f_{k} @ e w\right)$ for all fields $f_{k}$.

Proof. The pairs ins ${ }_{p} @ p$ and ins $q @ q$ are exactly those considered in Definition 8. We consider the most significant cases, from which the others can be derived similarly.

\section{ins $_{p}$ is a const $v$ and ins $q$ is not a catch}

(The case when $\operatorname{ins}_{q}$ is a catch does not exist, since const $v$ never throws any exception.) By Definition 8 there are $\operatorname{arcs} U_{i_{p}, j_{p}}$ in the constraints generated for program $P$. From the definition of const $v$ (Section 4), we have $\sigma_{p}=\langle l\|s\| \mu\rangle$ and $\sigma_{q}=\langle l \| v:$ : $s \| \mu\rangle$. Hence, by definition of solution (Definition 10), we have $\alpha\left(\sigma_{q}\right)\left(l_{k}\right)=\alpha\left(\sigma_{p}\right)\left(l_{k}\right) \subseteq S\left(l_{k} @ p\right) \subseteq S\left(l_{k} @ q\right)$ for every $0 \leq k<$ $i_{p}=i_{q}$. Similarly, $\alpha\left(\sigma_{q}\right)\left(s_{k}\right)=\alpha\left(\sigma_{p}\right)\left(s_{k}\right) \subseteq \mathcal{S}\left(s_{k} @ p\right) \subseteq \mathcal{S}\left(s_{k} @ q\right)$ for every $0 \leq k<j_{p}=j_{q}-1$. Moreover, when $v \in \mathbb{Z} \cup\{$ null $\}$, we have $\alpha\left(\bar{\sigma}_{q}\right)\left(s_{j_{q}-1}\right)=\emptyset \subseteq S\left(s_{j_{q}-1} @ q\right)$. The same is true when $v \in \mathbb{L}$ since, in that case, $\mu(v)$ cannot have any uninit fields because const $v$ is defined (Section 4$)$. Since $\sigma_{p}$ and $\sigma_{q}$ have the same memory $\mu_{p}$, we also have $\alpha\left(\sigma_{q}\right)\left(f_{k}\right)=\alpha\left(\sigma_{p}\right)\left(f_{k}\right) \subseteq S\left(f_{k} @ e w\right)$ for all fields $f_{k}$.

\section{ins $s_{p}$ is a catch $v$ and ins $q$ is not a catch}

(The case when ins $q$ is a catch does not exist, since catch never throws any exception.) By Definition 8 there $\operatorname{are} \operatorname{arcs} U_{i_{p}, j_{p}}$ in the constraints generated for program $P$. From the definition of catch (Section 4), we have $\sigma_{p}=\langle l \|$ top $\| \mu\rangle$ and $\sigma_{q}=\langle l \|$ top $\| \mu\rangle$. Hence, by definition of solution (Definition 10), we conclude that $\alpha\left(\sigma_{q}\right)\left(l_{k}\right)=\alpha\left(\sigma_{p}\right)\left(l_{k}\right) \subseteq \mathcal{S}\left(l_{k} @ p\right) \subseteq \mathcal{S}\left(l_{k} @ q\right)$ for every $0 \leq k<$ $i_{p}=i_{q}$. Similarly, $\alpha\left(\sigma_{q}\right)\left(s_{k}\right)=\alpha\left(\sigma_{p}\right)\left(s_{k}\right) \subseteq \mathcal{S}\left(s_{k} @ p\right) \subseteq S\left(s_{k} @ q\right)$ for every $0 \leq k<j_{p}=j_{q}$. Since $\sigma_{p}$ and $\sigma_{q}$ have the same memory $\mu_{p}$, we also have $\alpha\left(\sigma_{q}\right)\left(f_{k}\right)=\alpha\left(\sigma_{p}\right)\left(f_{k}\right) \subseteq S\left(f_{k} @ e w\right)$ for all fields $f_{k}$.

ins $_{p}$ is a store $x t$ and ins $_{q}$ is not a catch

(The case when ins $q$ is a catch does not exist, since store $x t$ never throws any exception.) From the definition of store $x t$ (Section 4), we have $\sigma_{p}=\langle l \|$ top $:: s \| \mu\rangle$ and $\sigma_{q}=\langle l[i \mapsto$ top $]\|s\| \mu\rangle$. By Definition 8 the constraints generated for program $P$ include the arcs $l_{k} @ p \rightarrow l_{k} @ q$ for every $0 \leq k<i_{p}$ with $k \neq x$, and arcs $s_{k} @ p \rightarrow s_{k} @ q$ for every $0 \leq k<j_{p}-1$. Hence, by definition of solution (Definition 10) we have $\alpha\left(\sigma_{q}\right)\left(l_{k}\right)=\alpha\left(\sigma_{p}\right)\left(l_{k}\right) \subseteq \mathcal{S}\left(l_{k} @ p\right) \subseteq$ $\mathcal{S}\left(l_{k} @ q\right)$ for every $0 \leq k<i_{p}=i_{q}, k \neq x$. Similarly, $\alpha\left(\sigma_{q}\right)\left(s_{k}\right)=$ $\alpha\left(\sigma_{p}\right)\left(s_{k}\right) \subseteq S\left(s_{k} @ p\right) \subseteq S\left(s_{k} @ q\right)$ for every $0 \leq k<j_{p}-1=j_{q}$. Moreover, there is an arc $s_{j_{p}-1} @ p \rightarrow l_{x} @ q$, so that $\alpha\left(\sigma_{q}\right)\left(l_{x}\right)=$ $\alpha\left(\sigma_{p}\right)\left(s_{j_{p}-1}\right) \subseteq \mathcal{S}\left(s_{j_{p}-1} @ p\right) \subseteq \mathcal{S}\left(l_{x} @ q\right)$. Since $\sigma_{p}$ and $\sigma_{q}$ have the same memory $\mu_{p}$, also $\alpha\left(\sigma_{q}\right)\left(f_{k}\right)=\alpha\left(\sigma_{p}\right)\left(f_{k}\right) \subseteq \mathcal{S}\left(f_{k} @ e w\right)$ for all fields $f_{k}$.

ins $_{p}$ is a new $\kappa$ and ins $q$ is not a catch
By Definition 8 there are arcs $U_{i_{p}, j_{p}}$ in the constraints generated for program $P$. From the definition of new $\kappa$ (Section 4), we have $\sigma_{p}=\langle l\|s\| \mu\rangle$ and $\sigma_{q}=\langle l\|\ell:: s\| \mu[\ell \mapsto o]\rangle$ (the other case of the definition of new $\kappa$ cannot be used here since ins $s_{q}$ is not a catch and hence it is only defined on non-exceptional states). We know that $o$ is an object of class $\kappa$ whose reference fields are all bound to uninit. Moreover, $\sigma_{p}$ and $\sigma_{q}$ have the same memory $\mu_{p}$, but for a fresh location $\ell$ (hence not yet reachable from the fields). Hence, by definition of solution (Definition 10), we conclude that $\alpha\left(\sigma_{q}\right)\left(l_{k}\right)=$ $\alpha\left(\sigma_{p}\right)\left(l_{k}\right) \subseteq S\left(l_{k} @ p\right) \subseteq S\left(l_{k} @ q\right)$ for every $0 \leq k<i_{p}=i_{q}$. Similarly, $\alpha\left(\sigma_{q}\right)\left(s_{k}\right)=\alpha\left(\sigma_{p}\right)\left(s_{k}\right) \subseteq \mathcal{S}\left(s_{k} @ p\right) \subseteq \mathcal{S}\left(s_{k} @ q\right)$ for every $0 \leq$ $k<j_{p}=j_{q}-1$. By definition of $o$ and since there is an arc $\left\{\kappa^{\prime} . f:\right.$ $t \mid t \in \mathbb{K}$ and $\left.\kappa \leq \kappa^{\prime}\right\} \rightarrow s_{j_{q}-1} @ q$, we have $\alpha\left(\sigma_{q}\right)\left(s_{j_{q}-1}\right)=\left\{\kappa^{\prime} \cdot f:\right.$ $t \mid t \in \mathbb{K}$ and $\left.\kappa \leq \kappa^{\prime}\right\} \subseteq \mathcal{S}\left(s_{j_{q}-1} @ q\right)$. We also have $\alpha\left(\sigma_{q}\right)\left(f_{k}\right)=$ $\alpha\left(\sigma_{p}\right)\left(f_{k}\right) \subseteq S\left(f_{k} @ e w\right)$ for all fields $f_{k}$.

\section{ins $p$ is a new $\mathrm{K}$ and ins $q$ is a catch}

This case exemplifies the situation when $\operatorname{ins}_{q}$ is a catch. Similar cases can be proved in the same way. By Definition 8 there are $\operatorname{arcs} U_{i_{p}, 0}$ in the constraints generated for program $P$. From the definition of new $\kappa$ (Section 4), we have $\sigma_{p}=\langle l\|s\| \mu\rangle$ and $\sigma_{q}=$ $\langle l\|\ell\| \mu[\ell \mapsto$ oome $]\rangle$ (the other case of the definition of new $\kappa$ cannot be used here since ins $s_{q}$ is a catch and hence it is only defined on exceptional states). We know that oome is an object of class OutofMemoryError without uninit fields. Moreover, $\sigma_{p}$ and $\sigma_{q}$ have the same memory $\mu_{p}$, but for a fresh location $\ell$ (hence not yet reachable from the fields). Hence, by definition of solution (Definition 10), we have $\alpha\left(\sigma_{q}\right)\left(l_{k}\right)=\alpha\left(\sigma_{p}\right)\left(l_{k}\right) \subseteq S\left(l_{k} @ p\right) \subseteq S\left(l_{k} @ q\right)$ for every $0 \leq k<i_{p}=i_{q}$. Moreover, $\alpha\left(\sigma_{q}\right)\left(s_{0}\right)=\emptyset \subseteq S\left(s_{0} @ q\right)$. We also have $\alpha\left(\sigma_{q}\right)\left(f_{k}\right)=\alpha\left(\sigma_{p}\right)\left(f_{k}\right) \subseteq \mathcal{S}\left(f_{k} @ e w\right)$ for all fields $f_{k}$.

$$
\text { ins }_{p} \text { is a getfield } f \text { and ins } q \text { is not a catch }
$$

By Definition 8 there are arcs $U_{i_{p}, j_{p}-1}$ in the constraints generated for program $P$. From the definition of getfield $f$ (Section 4), we have $\sigma_{p}=\langle l\|r e c:: s\| \mu\rangle$ and $\sigma_{q}=\langle l\|x:: s\| \mu\rangle$ with $x \in \mathbb{Z} \cup$ $\left\{\right.$ null,$\left.\mu_{p}(\mathrm{rec}) . f\right\}$ (the last case of the definition of getfield $f$ cannot be used here since ins $q$ is not a catch and hence it is only defined on non-exceptional states). Hence, by definition of solution (Definition 10), we have $\alpha\left(\sigma_{q}\right)\left(l_{k}\right)=\alpha\left(\sigma_{p}\right)\left(l_{k}\right) \subseteq \mathcal{S}\left(l_{k} @ p\right) \subseteq$ $S\left(l_{k} @ q\right)$ for every $0 \leq k<i_{p}=i_{q}$. Similarly we conclude that $\alpha\left(\sigma_{q}\right)\left(s_{k}\right)=\alpha\left(\sigma_{p}\right)\left(s_{k}\right) \subseteq S\left(s_{k} @ p\right) \subseteq S\left(s_{k} @ q\right)$ for every $0 \leq k<$ $j_{p}-1=j_{q}-1$. Moreover, we have $\alpha\left(\sigma_{q}\right)\left(s_{j_{q}-1}\right)=\emptyset$ when $x \in \mathbb{Z} \cup$ $\{$ null $\}$ and (Definition 7) $\alpha\left(\sigma_{q}\right)\left(s_{j_{q}-1}\right)=\left\{g \mid \mu_{p}(x) \cdot g=\right.$ uninit $\}$ when $x=\mu_{p}($ rec $) . f \in \mathbb{L}$. In the latter case, $\alpha\left(\sigma_{q}\right)\left(s_{j_{q}-1}\right) \subseteq\{g \mid$ there exists $\ell \in \mathbb{L}$ such that $\mu_{p}\left(\mu_{p}(\ell) . f\right) \cdot g=$ uninit $\}=\alpha\left(\sigma_{q}\right)(f)$. Since $\sigma_{p}$ and $\sigma_{q}$ have the same memory $\mu_{p}$, we conclude that $\alpha\left(\sigma_{q}\right)\left(s_{j_{q}-1}\right) \subseteq \alpha\left(\sigma_{q}\right)(f)=\alpha\left(\sigma_{p}\right)(f) \subseteq \mathcal{S}(f @ e w)$. By Definition 8, the constraints for program $P$ include the arc $f @ e w \rightarrow$ $s_{j_{p}-1} @ q$. Since $j_{p}=j_{q}$ and by definition of solution (Definition 10), we have $\alpha\left(\sigma_{q}\right)\left(s_{j_{q}-1}\right) \subseteq \mathcal{S}(f @ e w) \subseteq S\left(s_{j_{q}-1} @ q\right)$. Since $\sigma_{p}$ and $\sigma_{q}$ have the same memory $\mu_{p}$, we conclude that $\alpha\left(\sigma_{q}\right)\left(f_{k}\right)=$ $\alpha\left(\sigma_{p}\right)\left(f_{k}\right) \subseteq S\left(f_{k} @ e w\right)$ for all fields $f_{k}$.

$$
\text { ins }_{p} \text { is a putfield } f \text { and ins } q \text { is not a catch }
$$

From the definition of putfield $f$ (Section 4$), \sigma_{p}=\langle l \|$ top :: rec $::$ $s \| \mu\rangle$ and

$$
\sigma_{q}=\langle l\|s\| \underbrace{\mu[\mu(r e c) \cdot f \mapsto t o p]}_{\mu_{q}}\rangle
$$

(the last case of the definition of putfield $f$ cannot be used here since ins $q$ is not a catch and hence it is only defined on nonexceptional states). By Definition 8 there $\operatorname{are} \operatorname{arcs} l_{k} @ p \rightarrow l_{k} @ q$ 
in the constraints generated for $\operatorname{program} P$, for every $0 \leq k<i_{p}$ with $\left(l_{k}, s_{j_{p}-2}\right) \notin$ alias $_{p}$, and $\operatorname{arcs} l_{k} @ p \stackrel{f}{\rightarrow} l_{k} @ q$ for every $0 \leq k<i_{p}$ with $\left(l_{k}, s_{j_{p}-2}\right) \in$ alias $_{p}$. In the former case, since the fields of an object can only be initialized during the execution of this bytecode, by Definition 10 we conclude that $\alpha\left(\sigma_{q}\right)\left(l_{k}\right) \subseteq \alpha\left(\sigma_{p}\right)\left(l_{k}\right) \subseteq$ $S\left(l_{k} @ p\right) \subseteq \mathcal{S}\left(l_{k} @ q\right)$. In the latter case, by Definition 7 we have $\alpha\left(\sigma_{q}\right)\left(l_{k}\right)=\emptyset$ if $l[k] \notin \mathbb{L}$, and $\alpha\left(\sigma_{q}\right)\left(l_{k}\right)=\left\{g \mid \mu_{q}(l[k]) \cdot g=\right.$ uninit $\}$ if $l[k] \in \mathbb{L}$. Since we are assuming that $\left(l_{k}, s_{j_{p}-2}\right) \in$ alias $_{p}$, we know that $l[k]=$ rec. Since $\mu_{q}(r e c) . f=t o p \neq$ uninit (the value uninit is only allowed as the value of a field, never as a value on the stack), we conclude that $f \notin \alpha\left(\sigma_{q}\right)\left(l_{k}\right)=\left\{g \mid \mu_{q}(r e c) . g=\right.$ uninit $\}$. Since the fields of an object can only be initialized during the execution of this bytecode, by Definition 10 we conclude that, in this case, $\alpha\left(\sigma_{q}\right)\left(l_{k}\right) \subseteq \alpha\left(\sigma_{p}\right)\left(l_{k}\right) \backslash\{f\} \subseteq \mathcal{S}\left(l_{k} @ p\right) \backslash\{f\} \subseteq$ $\mathcal{S}\left(l_{k} @ q\right)$. The same proof can be applied to the stack elements. Given a field $f_{k} \neq f$, we have that $\mu_{p}$ and $\mu_{q}$ agree on the values of field $f_{k}$ of their objects. Hence $\alpha\left(\sigma_{q}\right)\left(f_{k}\right)=\alpha\left(\sigma_{p}\right)\left(f_{k}\right) \subseteq$ $\mathcal{S}\left(f_{k} @ e w\right)$. For field $f$, instead, we have (Definition 7) $\alpha\left(\sigma_{q}\right)(f)=$ $\left\{g \mid\right.$ there exists $\ell \in \mathbb{L}$ such that $\mu_{q}\left(\mu_{q}(\ell) . f\right) . g=$ uninit $\}$. Since $\mu_{q}(\ell) . f \in\left\{\mu_{p}(\ell) . f, t o p\right\}$ and $\mu_{q}(\ell)=\mu_{p}(\ell)$ for all locations $\ell$ where $\mu_{p}(\ell)$ (or, equivalently, $\mu_{q}(\ell)$ ) is defined, we have $\alpha\left(\sigma_{q}\right)(f) \subseteq\{g \mid$ there exists $\ell \in \mathbb{L}$ s.t. $\mu_{q}\left(\mu_{p}(\ell) . f\right) . g=$ uninit $\} \cup\left\{g \mid \mu_{p}(\right.$ top $) . g=$ uninit $\}=\left\{g \mid\right.$ there exists $\ell \in \mathbb{L}$ s.t. $\mu_{p}\left(\mu_{p}(\ell) . f\right) . g=$ uninit $\} \cup$ $\alpha\left(\sigma_{p}\right)\left(s_{j_{p}-1}\right)=\alpha\left(\sigma_{p}\right)(f) \cup \alpha\left(\sigma_{p}\right)\left(s_{j_{p}-1}\right)$. By Definition 8, we know that there is an arc $s_{j_{p}-1} @ p \rightarrow f @ e w$ among the arcs built for $P$. Thus $\alpha\left(\sigma_{q}\right)(f) \subseteq \alpha\left(\sigma_{p}\right)(f) \cup \alpha\left(\sigma_{p}\right)\left(s_{j_{p}-1}\right) \subseteq \mathcal{S}(f @ e w)$.

\section{A.1 Proof of Proposition 1}

Proposition 1, defined in Section 5.3, states that the analysis is safe: it never reports as non-raw a reference that may not be fully initialized. We now prove this proposition.

\section{Proof of Proposition 1}

We observe that the blocks in the configurations of an activation stack, but the topmost, cannot be both empty and with no successors. This is because configurations are only stacked by rule (2) at page 5 and if rest is empty there, then $m \geq 1$ or otherwise the code ends with a call bytecode and no return, which is illegal in Java bytecode [18].

We proceed by induction on the length $n$ of the execution

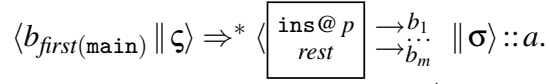

If $n=0$, the execution is just $\left\langle b_{\text {first (main) }} \| \varsigma\right\rangle$ and $\sigma=\varsigma$. Since the objects in the memory of $\zeta$ have no uninitialized fields, we have $\alpha(\sigma)\left(l_{k}\right)=\emptyset$ for every $0 \leq k<i, \alpha(\sigma)\left(s_{k}\right)=\emptyset$ for every $0 \leq k<j$ and $\alpha(\sigma)\left(f_{k}\right)=\emptyset$ for all fields $f_{k}$, so that the thesis holds.

Assume now that the thesis holds for any such execution of length $n^{\prime} \leq n$. Consider an execution

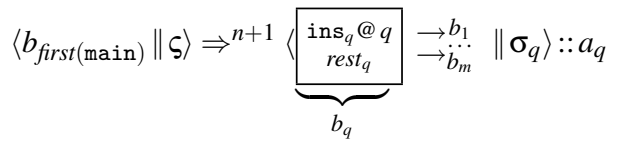

with $i n s_{q}\left(\sigma_{q}\right)$ defined. This execution must have the form

$$
\begin{aligned}
& \left\langle b_{\text {first(main) }} \| \varsigma\right\rangle \Rightarrow^{n_{p}}\langle\underbrace{\begin{array}{c}
\text { ins }_{p} @ p \\
\text { rest }_{p}
\end{array}}_{b_{p}} \rightarrow \rightarrow_{b_{m^{\prime}}^{\prime}}^{\prime} \| \sigma_{p}\rangle:: a_{p} \\
& \Rightarrow^{n+1-n_{p}}\left\langle b_{q} \| \sigma_{q}\right\rangle:: a_{q}
\end{aligned}
$$

with $0 \leq n_{p} \leq n$, that is, it must have a strict prefix of length $n_{p}$ whose final activation stack has a topmost configuration with a nonempty block $b_{p}$. This is because, for instance, $b_{\text {first(main) }}$ is a nonempty block (the main method must contain at least a return). Let hence such $n_{p}$ be maximal, $i_{p}$ be the number of local variables at $p, j_{p}$ the number of stack elements at $p$ and similarly $i_{q}$ and $j_{q}$ at $q$. By inductive hypothesis we know that

$$
\begin{gathered}
\alpha\left(\sigma_{p}\right)\left(l_{k}\right) \subseteq \mathcal{S}\left(l_{k} @ p\right) \quad \text { for all } 0 \leq k<i_{p} \\
\alpha\left(\sigma_{p}\right)\left(s_{k}\right) \subseteq S\left(s_{k} @ p\right) \quad \text { for all } 0 \leq k<j_{p} \\
\alpha\left(\sigma_{p}\right)\left(f_{k}\right) \subseteq \mathcal{S}\left(f_{k} @ e w\right) \text { for all fields } f_{k} .
\end{gathered}
$$

We will prove that (9) holds for $q$ instead of $p$ also, which completes the proof by induction. We distinguish on the basis of the rule of the operational semantics that is applied at the beginning of the derivation $\Rightarrow^{n+1-n_{p}}$ in Equation (8).

Rule (1). Then $\operatorname{ins}_{p}\left(\sigma_{p}\right)$ is defined and ins $p$ is not a call. case a: ins $s_{p}$ is not a return nor a throw

If rest $p$ is non-empty then, by the maximality of $n_{p},(8)$ must be

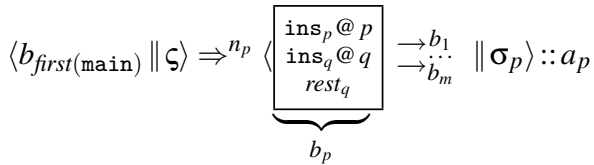

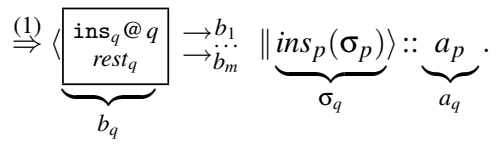

Otherwise $m^{\prime} \geq 1$ (legal Java bytecode can only end with a return or a throw) and, by the maximality of $n_{p}$, it must be $b_{q}=b_{h}^{\prime}$ for a suitable $1 \leq h \leq m^{\prime}$, so that (8) must have the form

$$
\begin{aligned}
& \left\langle b_{\text {first(main) }} \| \varsigma\right\rangle \Rightarrow^{n_{p}}\langle\underbrace{\underbrace{}_{\text {ins }_{p} @ p}}_{b_{p}} \rightarrow \underset{b_{m^{\prime}}^{\prime}}{b_{1}^{\prime}} \| \sigma_{p}\rangle:: a_{p}
\end{aligned}
$$

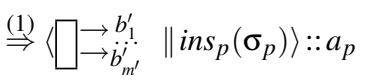

$$
\begin{aligned}
& \stackrel{(6)}{\Rightarrow}\langle b_{q} \| \underbrace{i n s_{p}\left(\sigma_{p}\right)}_{\sigma_{q}}\rangle:: \underbrace{a_{p}}_{a_{q}} .
\end{aligned}
$$

By the inductive hypothesis (9) for $n_{p}$ and Lemma 1, we conclude that (9) holds also with $q$ instead of $p$.

case b: ins $p$ is a return $t$

We show the case when $t \neq$ void, since the other is simpler (there is no return value to consider). Then rest $p$ is empty and $m^{\prime}=$ 0 (no code is executed after a return in legal Java bytecode, but the method terminates) and since $i s_{p}\left(\sigma_{p}\right) \in \Xi$ (definition of return $t),(8)$ must be in one of these two forms, depending on the emptiness of block $b$ in (4):

$$
\begin{aligned}
& \left\langle b_{\text {first(main) }} \| \varsigma\right\rangle
\end{aligned}
$$

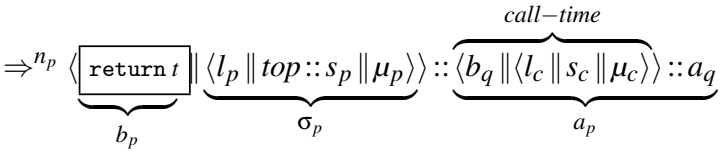

$$
\begin{aligned}
& \left.\stackrel{(1)}{\Rightarrow}\langle\bigsqcup|\left\langle l_{p} \| \text { top } \| \mu_{p}\right\rangle\right\rangle:: a_{p} \stackrel{(4)}{\Rightarrow}\langle b_{q} \| \underbrace{\left\langle l_{c} \| \text { top }:: s_{c} \| \mu_{p}\right\rangle}_{\sigma_{q}}\rangle:: a_{q}
\end{aligned}
$$

or

$$
\left\langle b_{\text {first(main) }} \| \varsigma\right\rangle
$$




$$
\begin{aligned}
& \Rightarrow^{n_{p}}\langle\underbrace{\langle\sqrt[\text { return } t]{ }|}_{b_{p}} \mid \underbrace{\left\langle l_{p} \| \text { top }:: s_{p} \| \mu_{p}\right\rangle}_{\sigma_{p}}\rangle:: \underbrace{\overbrace{\| \longrightarrow b_{1}^{\prime}}^{\text {call-time }} \|\left\langle l_{c}\left\|s_{c}\right\| \mu_{c}\right\rangle\rangle:: a_{q}}_{a_{p}} \\
& \left.\stackrel{(1)}{\Rightarrow}\langle\llbracket|\left\langle l_{p} \| \text { top } \| \mu_{p}\right\rangle\right\rangle:: a_{p} \stackrel{(4)}{\Rightarrow}\left\langle\square \underset{b_{m^{\prime}}}{\stackrel{b_{1}^{\prime}}{b^{\prime}}} \|\left\langle l_{c} \| \text { top }:: s_{c} \| \mu_{p}\right\rangle\right\rangle:: a_{q} \\
& \stackrel{(6)}{\Rightarrow}\langle b_{q} \| \underbrace{\left\langle l_{c} \| \text { top }:: s_{c} \| \mu_{p}\right\rangle}_{\sigma_{q}}\rangle:: a_{q}
\end{aligned}
$$

where, in the latter case, by maximality of $n_{p}$, we have $b_{q}=b_{h}^{\prime}$ for a suitable $1 \leq h \leq m^{\prime}$. We only prove the case for (10), the other being similar. Consider configuration call-time. Since only rule (2) can stack configurations, it was on top when a call was executed and (10) must have the form

$$
\begin{aligned}
& \left\langle b_{\text {first(main) }} \| \varsigma\right\rangle
\end{aligned}
$$

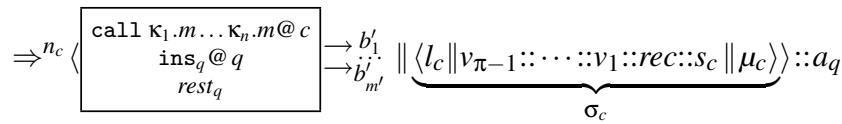

$$
\begin{aligned}
& \stackrel{(2)}{\Rightarrow}\left\langle\operatorname{first}\left(\kappa_{w} . m\right) \|\left\langle\left[\text { rec }:: v_{1}:: \cdots:: v_{\pi-1}\right]\|\varepsilon\| \mu_{c}\right\rangle\right\rangle:: a_{p} \\
& \left.\Rightarrow^{n_{p}-n_{c}-1}\left\langle b_{p} \| \sigma_{p}\right\rangle:: a_{p} \stackrel{(1)}{\Rightarrow}\langle\bigsqcup|\left\langle l_{p} \| \text { top } \| \mu_{p}\right\rangle\right\rangle:: a_{p} \stackrel{(4)}{\Rightarrow}\left\langle b_{q} \| \sigma_{q}\right\rangle:: a_{q}
\end{aligned}
$$

for a suitable $1 \leq w \leq n$, where the rules in the portion $\Rightarrow^{n_{p}-n_{c}-1}$ never make the stack lower than at the beginning of that portion. By inductive hypothesis for $n_{p}$ and $n_{c}$, we know that (9) holds for both $p$ and $c$. Since it holds for $p$ and the memory in $\sigma_{q}$ is $\mu_{p}$, we conclude that

$$
\alpha\left(\sigma_{q}\right)\left(f_{k}\right) \subseteq \mathcal{S}\left(f_{k} @ e w\right) \text { for all fields } f_{k} .
$$

Since it holds for $c$, since the fields of an object can only be initialized during the execution of the program (Section 4) and since $i_{c}=i_{q}$, we conclude that

$$
\begin{array}{ll}
\alpha\left(\sigma_{q}\right)\left(l_{k}\right) \subseteq \alpha\left(\sigma_{c}\right)\left(l_{k}\right) \subseteq \mathcal{S}\left(l_{k} @ c\right) & \text { for all } 0 \leq k<i_{q} \\
\alpha\left(\sigma_{q}\right)\left(s_{k}\right) \subseteq \alpha\left(\sigma_{c}\right)\left(s_{k}\right) \subseteq S\left(s_{k} @ c\right) & \text { for all } 0 \leq k<j_{q}-1 .
\end{array}
$$

By Definition 8, we have $\mathcal{S}\left(l_{k} @ c\right) \subseteq \mathcal{S}\left(l_{k} @ q\right)$ for all those $l_{k}$ for which an arc $l_{k} @ c \rightarrow l_{k} @ q$ is built (similarly for $s_{k}$ ). For them we have $\alpha\left(\sigma_{q}\right)\left(l_{k}\right) \subseteq \mathcal{S}\left(l_{k} @ q\right)$ and $\alpha\left(\sigma_{q}\right)\left(s_{k}\right) \subseteq \mathcal{S}\left(s_{k} @ q\right)$. For the $l_{k}$ that, instead, at call-time, are definite alias of an actual parameter $s_{j_{c}-u-1}$ of the call and such that the corresponding formal parameter $l_{\pi-u-1}$ is never modified inside $\kappa_{w} \cdot m$, by inductive hypothesis for $n_{p}$ we know that

$$
\alpha\left(\sigma_{q}\right)\left(l_{k}\right)=\alpha\left(\sigma_{p}\right)\left(l_{\pi-u-1}\right) \subseteq \mathcal{S}\left(l_{\pi-u-1} @ p\right)
$$

(similarly for $s_{k}$ ). By Definition 8 , there is an arc of the form $l_{\pi-u-1} @$ end of $\kappa_{w} \cdot m \rightarrow l_{k} @ q$ among the constraints for program

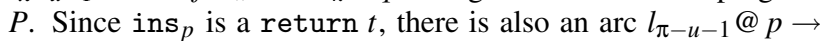
$l_{\pi-u-1} @$ end of $\kappa_{w} . m$. Hence

$\alpha\left(\sigma_{q}\right)\left(l_{k}\right) \subseteq \mathcal{S}\left(l_{\pi-u-1} @ p\right) \subseteq \mathcal{S}\left(l_{\pi-u-1} @\right.$ end of $\left.\kappa_{w} \cdot m\right) \subseteq \mathcal{S}\left(l_{k} @ q\right)$ and similarly for $s_{k}$ with $0 \leq k<s_{j_{q}-1}$. It remains to prove the same result for $s_{j_{q}-1}$, that is, for the returned value. By inductive hypothesis for $n_{p}$ we know that $\alpha\left(\sigma_{p}\right)\left(s_{j_{p}-1}\right) \subseteq \mathcal{S}\left(s_{j_{p}-1} @ p\right)$. By Definition 8, there are arcs $s_{j_{p}-1} @ p \rightarrow$ return@ $\kappa_{w} . m$ and return@ $\kappa_{w} \cdot m \rightarrow s_{j_{c}-\pi} @ q$. Moreover, $j_{c}=j_{q}-1+\pi$ so that $j_{c}-\pi=j_{q}-1$. We conclude that

$$
\begin{aligned}
& \alpha\left(\sigma_{q}\right)\left(s_{j_{q}-1}\right)=\alpha\left(\sigma_{p}\right)\left(s_{j_{p}-1}\right) \subseteq \mathcal{S}\left(s_{j_{p}-1} @ p\right) \\
& \quad \subseteq \mathcal{S}\left(\text { return@ } \kappa_{w} . m\right) \subseteq \mathcal{S}\left(s_{j_{c}-\pi} @ q\right)=\mathcal{S}\left(s_{j_{q}-1} @ q\right) .
\end{aligned}
$$

case c: ins $p$ is a throw $\kappa$

If rest $p$ is empty and $m^{\prime}>0$, the execution (8) must have the form

$$
\begin{aligned}
& \left\langle b_{\text {first (main) }} \| \varsigma\right\rangle \Rightarrow^{n_{p}}\langle\underbrace{\underbrace{}_{\text {throw } \kappa}}_{b_{p}} \rightarrow b_{b_{m^{\prime}}^{\prime}}^{b_{1}^{\prime}} \| \underbrace{\left.\left\langle l_{p}\left\|e:: s_{p}\right\| \mu_{p}\right\rangle\right\rangle}_{\sigma_{p}}:: a_{p} \\
& \stackrel{(1)}{\Rightarrow}\langle\square \rightrightarrows \dot{b}_{\dot{b}_{m^{\prime}}^{\prime}}^{\prime} \quad \| \underbrace{\left\langle l_{p}\|e\| \mu_{p}\right\rangle}_{\sigma_{q}}\rangle:: a_{p} \stackrel{(6)}{\Rightarrow}\left\langle b_{q} \| \sigma_{q}\right\rangle:: \underbrace{a_{p}}_{a_{q}}
\end{aligned}
$$

where, by maximality of $n_{p}$, we have $b_{q}=b_{h}^{\prime}$ for a suitable $1 \leq$ $h \leq m^{\prime}$. If rest $t_{p}$ is non-empty, the execution (8) must have the form

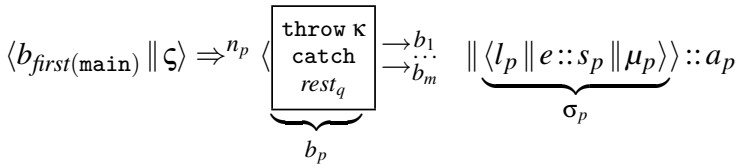

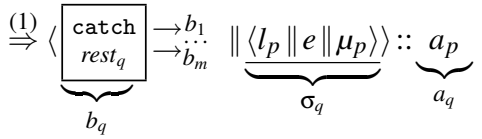

since catch is the only bytecode whose semantics can be defined on the exceptional state $\sigma_{q}$. In both these cases, by inductive hypothesis for $n_{p}$ and Lemma 1 , we have the thesis.

If rest $t_{p}$ is empty and $m^{\prime}=0$, the execution (8) must have one of these two forms, depending on the emptiness of block $b$ in (5):

$$
\begin{aligned}
& \left\langle b_{\text {first(main) }} \| \varsigma\right\rangle
\end{aligned}
$$

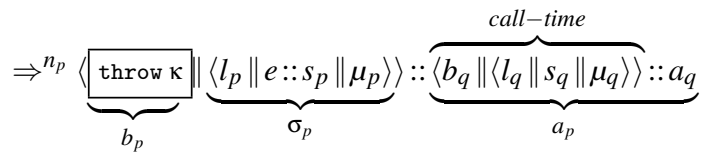

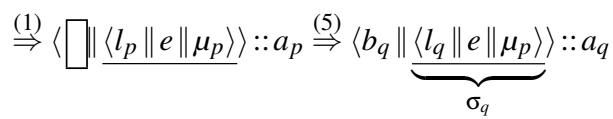

or

$$
\begin{aligned}
& \left\langle b_{\text {first(main) }} \| \varsigma\right\rangle
\end{aligned}
$$

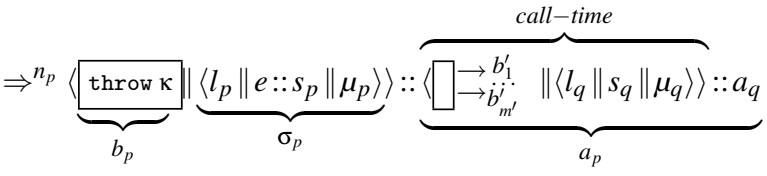

$$
\begin{aligned}
& \stackrel{(1)}{\Rightarrow}\langle\square \underline{\left.\left\langle l_{p}\|e\| \mu_{p}\right\rangle\right\rangle:: a_{p} \stackrel{(5)}{\Rightarrow}\left\langle\square \rightrightarrows \dot{b}_{m^{\prime}}^{\prime}\right.} \| \underbrace{\left.\left.\left\|l_{q}\right\| e \| \mu_{p}\right\rangle\right\rangle}_{\sigma_{q}}:: a_{q} \\
& \stackrel{(6)}{\Rightarrow}\langle b_{q} \| \underbrace{\left\langle l_{q}\|e\| \mu_{p}\right\rangle}_{\sigma_{q}}\rangle:: a_{q}
\end{aligned}
$$

where, by maximality of $n_{p}$, we have $b_{q}=b_{h}^{\prime}$ for a suitable $1 \leq$ $h \leq m^{\prime}$. We only prove the case (11), the other being similar. Consider configuration call-time. Since only rule (2) can stack configurations, it was on top when a call was executed and (11) must have the form

$$
\begin{aligned}
& \left\langle b_{\text {first(main) }} \| \varsigma\right\rangle
\end{aligned}
$$

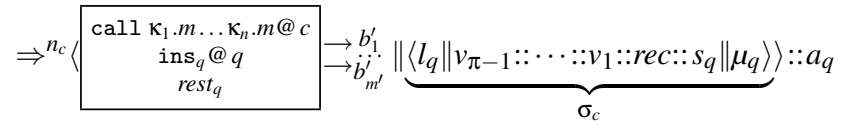




$$
\begin{aligned}
& \stackrel{(2)}{\Rightarrow}\left\langle\text { first }\left(\kappa_{w} \cdot m\right) \|\left\langle\left[r e c:: v_{1}:: \cdots:: v_{\pi-1}\right]\|\varepsilon\| \mu_{q}\right\rangle\right\rangle::\left\langle b_{q} \|\left\langle l_{q}\left\|s_{q}\right\| \mu_{q}\right\rangle\right\rangle:: a_{q}
\end{aligned}
$$

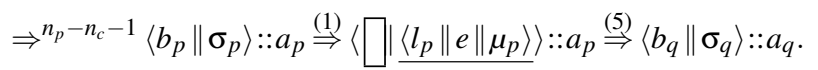

Since $\operatorname{ins}_{q}\left(\sigma_{q}\right)$ is defined and $\sigma_{q} \in \underline{\Xi}$, the only possibility is that ins $q$ is a catch (it is the only bytecode defined on exceptional states). By Definition 8, the constraints for program $P$ must include the arcs $l_{k} @ c \rightarrow l_{k} @ q$. By inductive hypothesis (9) for $n_{c}$, we know that $\alpha\left(\sigma_{c}\right)\left(l_{k}\right) \subseteq \mathcal{S}\left(l_{k} @ c\right)$ for $0 \leq k<i_{c}=i_{q}$. Since the fields of an object can only be initialized during the execution of the program, by inductive hypothesis for $n_{c}$ we conclude that $\alpha\left(\sigma_{q}\right)\left(l_{k}\right) \subseteq \alpha\left(\sigma_{c}\right)\left(l_{k}\right) \subseteq \mathcal{S}\left(l_{k} @ c\right) \subseteq \mathcal{S}\left(l_{k} @ q\right)$ (because of the arc $\left.l_{k} @ c \rightarrow l_{k} @ q\right)$. Consider now $s_{0}=e$, the only stack element of $\sigma_{q}$. We have $\alpha\left(\sigma_{q}\right)\left(s_{0}\right)=\alpha\left(\sigma_{p}\right)\left(s_{j_{p}-1}\right) \subseteq \mathcal{S}\left(s_{j_{p}-1} @ p\right)$ (by inductive hypothesis for $n_{p}$ ). By Definition 8 , there are arcs $s_{j_{p}-1} @ p \rightarrow$ exception@ $\kappa_{w} . m$ and exception@ $\kappa_{w} . m \rightarrow s_{0} @ q$ in the constraints for program $P$. Then $\alpha\left(\sigma_{q}\right)\left(s_{0}\right) \subseteq \mathcal{S}\left(s_{j_{p}-1} @ p\right) \subseteq$ $S\left(\right.$ exception@ $\left.\kappa_{w} \cdot m\right) \subseteq S\left(s_{0} @ q\right)$. Finally, by inductive hypothesis for $n_{p}$ we know that $\alpha\left(\sigma_{p}\right)\left(f_{k}\right) \subseteq \mathcal{S}\left(f_{k} @ e w\right)$ for all fields $f_{k}$. Since the memory of $\sigma_{q}$ is still $\mu_{p}$, we conclude that $\alpha\left(\sigma_{q}\right)\left(f_{k}\right) \subseteq$ $S\left(f_{k} @ e w\right)$ for all fields $f_{k}$.

Rule (2). By definition of makescope, (8) must have the form

$$
\begin{aligned}
& \left\langle b_{\text {first(main) }} \| \varsigma\right\rangle \Rightarrow^{n_{p}}
\end{aligned}
$$

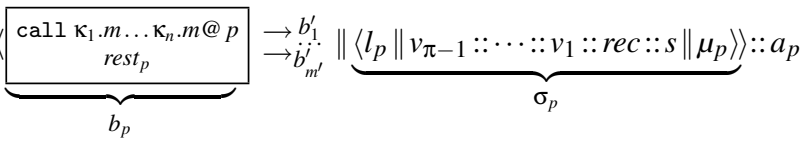

$$
\begin{aligned}
& \stackrel{(2)}{\Rightarrow}\langle\underbrace{\operatorname{first}\left(\kappa_{i} . m\right)}_{b_{q}} \| \underbrace{\left\langle\left[r e c:: v_{1}:: \cdots:: v_{\pi-1}\right]\|\varepsilon\| \mu_{p}\right\rangle}_{\sigma_{q}}\rangle:: a_{q} .
\end{aligned}
$$

Since $\sigma_{p}$ and $\sigma_{q}$ have the same memory $\mu_{p}$, for the shape of their stack and local variable array we have $\alpha\left(\sigma_{q}\right)\left(l_{\pi-u-1}\right)=$ $\alpha\left(\sigma_{p}\right)\left(s_{j_{p}-u-1}\right)$ for every $0 \leq u<\pi$. By Definition 8 , the constraints for program $P$ include the arcs $s_{j_{p}-u-1} @ p \rightarrow l_{\pi-u-1} @ q$, since $q$ is the program point at the beginning of block first $\left(\kappa_{i} \cdot m\right)$. By inductive hypothesis for $n_{p}$ we conclude that

$\alpha\left(\sigma_{q}\right)\left(l_{\pi-u-1}\right)=\alpha\left(\sigma_{p}\right)\left(s_{j_{p}-u-1}\right) \subseteq \mathcal{S}\left(s_{j_{p}-u-1} @ p\right) \subseteq \mathcal{S}\left(l_{\pi-u-1} @ q\right)$

for every $0 \leq u<\pi$. Since $i_{q}=\pi$, we conclude that

$$
\alpha\left(\sigma_{q}\right)\left(l_{k}\right) \subseteq \mathcal{S}\left(l_{k} @ q\right)
$$

for every $0 \leq k<i_{q}$. The same result for $s_{k}$ is vacuously true since $j_{q}=0$. For the fields, we observe that $\sigma_{p}$ and $\sigma_{q}$ have the same memory $\mu_{p}$. From the inductive hypothesis (9) for $n_{p}$, we conclude that $\alpha\left(\sigma_{q}\right)\left(f_{k}\right)=\alpha\left(\sigma_{p}\right)\left(f_{k}\right) \subseteq \mathcal{S}\left(f_{k} @ e w\right)$ for all fields $f_{k}$.

Rule (3). Then (8) must have the form

$$
\begin{aligned}
& \left\langle b_{\text {first(main) }} \| \varsigma\right\rangle \Rightarrow^{n_{p}} \\
& \underbrace{\left\langle\begin{array}{c}
\text { call } \kappa_{1} . m \ldots \kappa_{n} . m @ p \\
\text { rest } t_{p}
\end{array}\right.}_{b_{p}} \rightarrow \underset{b_{1}^{\prime}}{\rightarrow}\left\|\dot{b}_{m^{\prime}}^{\prime}\right\| \underbrace{\left\langle l_{p}\left\|v_{\pi-1}:: \cdots:: v_{1}:: \operatorname{null}:: s\right\| \mu_{p}\right\rangle}_{\sigma_{p}}\rangle:: a_{p} \\
& \stackrel{(3)}{\Rightarrow}\langle\underbrace{\sqrt[r e s t_{p} \longrightarrow b_{m^{\prime}}^{\prime} \rightarrow]{\rightarrow}}_{b_{q}} \| \underbrace{\left\langle b_{m^{\prime}}^{\prime}\|\ell\| \mu_{p}[\ell \mapsto n p e]\right\rangle}_{\sigma_{q}}\rangle: a_{q}
\end{aligned}
$$

when rest $p$ is non-empty, while otherwise it has the form

$$
\left\langle b_{\text {first(main) }} \| \varsigma\right\rangle \Rightarrow^{n_{p}}
$$

$$
\begin{aligned}
& \underbrace{\left\langle\text { call } \kappa_{1} . m \ldots \kappa_{n} . m @ p\right.}_{b_{p}} \rightarrow b_{m^{\prime}}^{b_{1}^{\prime}} \| \underbrace{\left\langle l_{p}\left\|v_{\pi-1}:: \cdots:: v_{1}:: \operatorname{null}:: s\right\| \mu_{p}\right\rangle}_{\sigma_{p}}\rangle:: a_{p} \\
& \stackrel{(3)}{\Rightarrow}\langle\bigsqcup \rightrightarrows \dot{b}_{m^{\prime}}^{\prime} \quad \| \underbrace{\left\langle l_{p}\|\ell\| \mu_{p}[\ell \mapsto n p e]\right\rangle}_{\sigma_{q}}\rangle:: a_{q} \stackrel{(6)}{\Rightarrow}\left\langle b_{q} \| \sigma_{q}\right\rangle:: a_{q}
\end{aligned}
$$

where, by maximality of $n_{p}$, we have $b_{q}=b_{h}^{\prime}$ for a suitable $1 \leq$ $h \leq m^{\prime}$. In both cases, we know that $\ell$ is fresh and npe is a NullPointerException object with no uninitialized fields. Moreover, $\sigma_{q} \in \underline{\Xi}$ and hence ins $q$ must be a catch (it is the only bytecode that is defined on an exceptional state). By Definition 8, there are arcs $l_{k} @ p \rightarrow l_{k} @ q$ for $0 \leq k<i_{p}=i_{q}$. We also know that $\sigma_{p}$ and $\sigma_{q}$ have the same memory $\mu_{p}$. By inductive hypothesis (9) for $n_{p}$, we have

$$
\alpha\left(\sigma_{q}\right)\left(l_{k} @ q\right)=\alpha\left(\sigma_{p}\right)\left(l_{k} @ p\right) \subseteq S\left(l_{k} @ p\right) \subseteq S\left(l_{k} @ q\right) .
$$

The state $\sigma_{q}$ has only one stack element $\ell$. Since npe has no uninitialized fields, we have $\alpha\left(\sigma_{q}\right)\left(s_{0}\right)=\emptyset \subseteq \mathcal{S}\left(s_{0} @ q\right)$. Since $\sigma_{p}$ and $\sigma_{q}$ have the same memory $\mu_{p}$ and by inductive hypothesis for $n_{p}$, we have $\alpha\left(\sigma_{q}\right)\left(f_{k}\right)=\alpha\left(\sigma_{p}\right)\left(f_{k}\right) \subseteq S\left(f_{k} @ e w\right)$ for all fields $f_{k}$.

Rules (4), (5) and (6). They cannot be applied since $b_{p}$ is nonempty. 